\title{
Fleißrevolution in Sachsen (17.-19. Jahrhundert)? Quellenproblematik und Methode der Erfassung historischer Zeitbudgets mittels Strafprozessakten
}

\author{
von \\ ANNE PURSCHWITZ und JOHANNA RIESE
}

Eine seit zwei Jahrzehnten in der Wirtschafts- und Sozialgeschichte häufig diskutierte Frage ist die nach der Zeitverwendung beziehungsweise den Arbeitszeiten in der Vergangenheit. Insbesondere für das Nicht-Statistische Zeitalter rückt die Erstellung, Rekonstruktion und Analyse von Arbeitszeitbudgets zunehmend in den Blick. Bahnbrechend in dieser Hinsicht sind die Studien von Hans-Joachim Voth ${ }^{1}$ und Jan de Vries $^{2}$ sowie auch Gregory Clark/Ysbrand van de Werf ${ }^{3}$ oder Roman Sandgruber, ${ }^{4}$ die mit durchaus konträren Methoden versuchen in diesem Bereich belastbare Ergebnisse zu gewinnen. Die betreffenden Studien stellen die bisher verbreitete Auffassung von der ,Mußepräferenz' in der Frühen Neuzeit in Frage und werfen einen neuen Blick auf den Themenkomplex Fleiß, Muße und Arbeitsintensität. Eine ähnliche Absicht verfolgt ein in Vorbereitung befindliches Projekt mit dem Titel: ,Fleißrevolution in Sachsen (17.-19. Jahrhundert)?‘. Um die Methodik des Projektes, die sich an dem 1997 erstmals von Hans-Joachim Voth in die historische Forschungspraxis eingeführten Verfahren der Erhebung historischer Zeitbudgets orientiert, und um die Machbarkeit auf Basis sächsischer Akten zu überprüfen, wurde eine stichprobenartige Erhebung und Analyse historischer Zeitbudgets durchgeführt. Dafür wurden die in Strafprozessakten enthaltenen Verhörprotokolle im Hinblick auf Angaben über Tages- und Zeitabläufe ausgewertet. Verhörprotokolle halten Aussagen von Zeugen oder Angeklagten fest und liegen grundsätzlich in den Prozessakten aller Zweige der Justiz vor. Eine besondere Dichte von Zeugenaussagen findet sich in den Unterlagen der Strafjustiz.

Basierend auf der Analyse der relevanten Forschungsliteratur und von Verhörprotokollen in ca. 80 sächsischen Strafprozessakten des Zeitraums zwischen 1680 und

1 Hans-Jonchim Voth, Time and Work in England, 1750-1830, Oxford 2001; Ders., Seasonality of conceptions as a source for historical time-budget analysis: tracing the disappearance of holy days in early modern England, in: Historical Methods 27 (1994), S. 127-132.

2 Jan DE VRIEs, The Industrious Revolution: Consumer Behavior and the Household Economy, 1650 to the Present, Cambridge 2008; Ders., The Industrial Revolution and the Industrious Revolution, in: Journal of Economic History 54 (1994), S. 249-270; Ders., Between Purchasing Power and the World of Goods: Understanding the Household Economy in Early Modern Europe, in: John Brewer/Roy Porter (Hg.), Consumption and the World of Goods, London 1993, S. 85-132.

3 Gregory Clark/Ysbrand van der Werf, Work in Progress? The Industrious Revolution, in: Journal of Economic History 58 (1998), S. 830-843.

4 Roman Sandgruber, Zeit der Mahlzeit. Veränderungen in Tagesablauf und Mahlzeiteneinteilung in Österreich im 18. und 19. Jahrhundert, in: Nils-Arvid Bringéus (Hg.), Wandel der Volkskultur in Europa. Festschrift für Günter Wiegelmann (Beiträge zur Volkskultur in Nordwestdeutschland 60), Bd. 1, Münster 1988, S. 459-472. 
1870 konnten folgende Untersuchungsgegenstände spezifiziert werden: 1. der Quellenwert und die -problematik von Verhörprotokollen, 2. die Anzahl der zu erfassenden Verhördaten unter Berücksichtigung der anzuwendenden Methoden, 3. die Quellenlage und erste Ergebnisse der Datenerfassung sowie 4. Schlussfolgerungen für die Implementierung der Methode in einer weiterführenden Studie.

\section{Quellenwert und -problematik von Verbörprotokollen \\ 1. Entstehungsgeschichte und Struktur der Strafgerichtsakten}

Ab dem Spätmittelalter bemühten sich die Städte aus praktischen Gründen um eine zunehmende Fixierung von Rechts- und Verwaltungsvorgängen. Strafgerichtsakten aus dieser Zeit sind jedoch nur spärlich überliefert. Mit Einführung des Inquisitionsprozesses und verstärkt in der Frühen Neuzeit kam es zu einer Zentralisierung der Kriminaljustiz in den Territorien. Die Gerichtsakten sind in der Lage, in einer gewissen Breite Delinquenz und Sanktionen zu bekunden. ${ }^{5}$ Vom 17. bis 19. Jahrhundert stellen Strafprozessakten ausführliche schriftliche Dokumentationen vergangenen Prozessgeschehens dar und sind in recht großem Umfang erhalten. Die Akten geben Auskunft über den Verlauf des Prozesses und die Beweisfindung vor Gericht. Sie enthalten, meist in chronologischer Reihenfolge, die Anzeige des Straftatbestandes, die den Prozess einleitenden Texte mit Informationen zu Tathergang und Beschuldigten, häufig gefolgt von Verhören und Befragungen von Anzeigenden, Zeugen und Tatverdächtigen, sowie oftmals Schriften von Verteidigern, den Schriftverkehr zwischen Behörden und das Urteil mit ausführlicher Urteilsbegründung, ergänzt um Berichte zur Urteilsvollstreckung. Den eigentlichen Verhandlungsakten sind zahlreiche weitere schriftliche Dokumente beigefügt, so beispielsweise Testamente, Verträge, Bittgesuche von Verwandten oder Bekannten aus dem sozialen Umfeld sowie Gutachten von Sachverständigen (z. B. von Ärzten oder den juristischen Fakultäten ${ }^{6}$ ). Die Strafgerichtsakten können dadurch sehr umfangreich sein, vor allem wenn in einem Prozess höhere Gerichtsinstanzen mit involviert wurden. Grundlegend bieten sie die Möglichkeit, „wesentliche Stationen aller Malefizverfahren von der Verhaftung bis zum Endurteil nachvollziehbar" werden zu lassen.7

Trotz ihrer Jahrhunderte währenden Entwicklung an Gerichten unterschiedlicher rechtlicher und räumlicher Zuständigkeit seit der Mitte des 13. Jahrhunderts weisen die Prozessakten des deutschen Sprachraums und besonders die beinhalteten Verhörprotokolle strukturelle Gemeinsamkeiten auf. Eine Ursache hierfür liegt in der weiten Verbreitung des römisch-kanonischen Verfahrensrechts seit dem Mittelalter. Es existierten detaillierte Regeln für die Auswahl der Zeugen, ihre Befragung und die

5 Vgl. Gerd Schwerhoff, Aktenkundig und gerichtsnotorisch. Einführung in die historische Kriminalitätsforschung (Historische Einführungen 3), Tübingen 1999, S. 25-35.

6 Diese Praxis war mit der Einführung der, Carolina' im Jahr 1532 verordnet worden. Constitutio Criminalis Carolina: Keyser Karls des fünfften: vnnd des heyligen Römischen Reichs peinlich gerichtsordnung/auff den Reichstägen zu Augspurgk vnd Regenspurk/inn jaren dreissig, vnn zwey vnd dreisssig gehalten/auffgericht und beschlossen, Mainz 1533, $\$ 219$ (Verzeichnis der im deutschen Sprachbereich erschienenen Drucke des 16. Jahrhunderts (VD 16): D 1069).

7 Vgl. Wolfagang Behringer, Mörder, Diebe, Ehebrecher. Verbrechen und Strafen in Kurbayern vom 16. bis 18. Jahrhundert, in: Richard van Dülmen (Hg.), Verbrechen, Strafen und soziale Kontrolle, Frankfurt a. M. 1990, S. 85-132, 287-293, hier S. 89. 
anschließende Bewertung ihrer Aussagen. ${ }^{8}$ Die deutschlandweite Strafprozessreform von 1849 brachte mit der Abkehr vom Inquisitionsprozess auch eine veränderte Dokumentationspraxis mit sich. Der Verhandlungsverlauf wurde nun meist knapper geschildert; Aussagen von Zeugen und Angeklagten finden sich oft an ungewohnter Stelle in der Akte wieder, sind aber immer noch in ausreichender Dichte vorhanden.

Die Protokolle der Verhöre sind teilweise als umfassende Fragekataloge angelegt und beschränken sich nicht nur auf den engeren Gegenstand des verhandelten Falles (,Interrogatoria specialia'), sondern zielen auf die ausführliche Dokumentation der aussagenden Person und ihrer persönlichen Umstände (,Interrogatoria generalia'). Um die Qualität der Zeugenaussagen bewerten zu können, waren die Richter angehalten, den Befragten „auf den Zahn zu fühlen“. ${ }^{9}$ Über den Betrachtungszeitraum von 1680 bis 1870 hinweg existieren zwei Varianten in der Verschriftlichung der Befragung: 1. artikulierte Verhöre, die sowohl die Fragen als auch die Aussagen wörtlich (wenn auch meist in indirekter Rede) wiedergeben und 2. summarische Verhöre, die eine Zusammenfassung der Aussage einer, in Einzelfällen auch mehrerer Personen bieten. Grundsätzlich weisen beide Typen relevante Angaben für die Untersuchung auf und können gleichberechtigt einbezogen werden.

\section{Gerichtsakten und Verhörprotokolle als Quelle in der Geschichtswissenschaft}

Gerichtsakten sind in der deutschsprachigen Forschungslandschaft seit dem 19. Jahrhundert als Quelle in der traditionellen Rechtsgeschichte, einer Teildisziplin der Rechtswissenschaften, verwendet worden, um die juristischen Aspekte staatlichen Handelns sowie die Entwicklung gesetzlicher Normen zu untersuchen. ${ }^{10}$ Im Zusammenhang mit der Strafrechtsgeschichte standen Aspekte der Ausprägung und Veränderung von Rechtsnormen, der Rechtspraxis und der Normierungsintensität im Vordergrund. Sozialhistorische Fragestellungen werden in diesen Analysen nur selten befriedigend beantwortet. ${ }^{11}$ Neben der Rechtsgeschichte bediente sich die ältere Kulturgeschichte um 1900 der Gerichtsakten. Sie entwickelte jedoch nur sehr bedingt analytische Instrumente für den Umgang mit den Quellen. ${ }^{12}$ In Abgrenzung zu dieser Forschungstradition etablierte sich in Deutschland ab den 1980er-Jahren die histori-

8 Ralf-Peter Fuchs/Winfried Schulze, Zeugenverhöre als historische Quellen. Einige Vorüberlegungen, in: Dies. (Hg.), Wahrheit, Wissen, Erinnerung (Wirklichkeit und Wahrnehmung in der Frühen Neuzeit 1), Münster 2002, S. 7-40, hier S. 22 f.

9 Ebd., S. 23.

10 Ausführlich zur Entwicklung der Rechts- und Kriminalitätsgeschichte vgl. SchwerHOFF, Aktenkundig und gerichtsnotorisch (wie Anm. 5), S. 15-23; Ders., Kriminalitätsgeschichte im deutschen Sprachraum. Zum Profil eines, verspäteten' Forschungszweiges, in: Ders./Andreas Blauert (Hg.), Kriminalitätsgeschichte. Beiträge zur Sozial- und Kulturgeschichte der Vormoderne, Konstanz 2000, S. 21-68; JoACHIM Eıвасн, Kriminalitätsgeschichte zwischen Sozialgeschichte und historischer Kulturforschung, in: Historische Zeitschrift 263 (1996), S. 681-715; Dirk Blasius, Kriminologie und Geschichtswissenschaft. Bilanz und Perspektiven interdisziplinärer Forschung, in: Geschichte und Gesellschaft 14 (1988), S. 136-149.

11 Vgl. dazu beispielsweise HinRICH RüPING, Grundriss der Strafrechtsgeschichte (Schriftenreihe der Juristischen Schulung 73), München 1981.

12 Theodor Hampe, Die Nürnberger Malefizbücher als Quellen der reichsstädtischen Sittengeschichte vom 14. bis zum 18. Jahrhundert, Bamberg 1927. 
sche Kriminologie beziehungsweise Kriminalitätsgeschichte. ${ }^{13}$ Ihre Studien basieren methodisch sowohl auf qualitativen als auch auf quantitativen Ansätzen. Vertreter der Kriminalitätsgeschichte fokussieren die Straf- und Gerichtspraxis sowie die lebensweltliche Perspektive der historischen Akteure. Sie arbeiten beispielsweise die Bedeutung unterschiedlicher Delikte heraus, ebenso die sozialen und ökonomischen Verhältnisse der Kriminellen sowie auch die städtischen und dörflichen Gegebenheiten, in denen sich Kriminalität abspielte. ${ }^{14}$ Weitergehende Forschungen weisen über die Thematisierung von Kriminalität und Konflikt im engen Sinne hinaus. Sie können als Zeugnisse von Arbeitsverhältnissen, Migrationsbewegungen oder Familienbeziehungen gelesen und ausgewertet werden. ${ }^{15}$

\section{Quellenproblematik und ihre Berücksichtigung}

Beim Einsatz der Time-Budget-Methode, die Voth der soziologischen Forschungspraxis entlehnt hat und bei der mittels Interviews Tagesabläufe rekonstruiert werden, müssen die Gütekriterien empirischer Untersuchungen erfüllt sein. Die Erhebung historischer Zeitbudgets hat jedoch nicht nur diesen Anforderungen zu genügen sowie spezifische Herausforderungen aufgrund des vor einigen Jahrhunderten entstandenen Untersuchungsmaterials zu berücksichtigen, sondern auch der Quellenkritik, die von der Geschichtswissenschaft eingefordert wird, Stand zu halten. Die in der Forschungsliteratur von Kriminalitätshistorikern wie von Kritikern der quantitativ-statistischen Untersuchung Voths diskutierten Hinweise zur Quellenproblematik werden im Folgenden angeführt und auf ihre Bedeutung für die Erfassung historischer Zeitbudgets im sächsischen Raum hin untersucht.

\section{a) Zwangskontext der Justiz}

Vertreter der Kriminalitätsgeschichte mahnen eine intensive Reflexion im Umgang mit den Quellen an, wenn in hermeneutischer Tradition qualitative Methoden für die Interpretation der Aussagen in Verhörprotokollen herangezogen werden. ${ }^{16}$ Es werden besonders Einschränkungen im Aussagewert der Quellen bezüglich der internen Validität der gemachten Angaben hervorgehoben. Die Angeklagten und Zeugen standen oftmals unter psychischem und physischem Druck, nicht selten bis hin zur Folter. Es sei davon auszugehen, dass die Aussagen durch Brechungen, Perspektivierungen und bewusste Verfälschungen geprägt sind. ${ }^{17}$ Die Problematik möglicher falscher Angaben in den Verhören kann jedoch in der vorliegenden Studie auf verschiedenen Ebenen

13 Vgl. Eibach, Kriminalitätsgeschichte (wie Anm. 10); André Krischer, Neue Forschungen zur Kriminalitätsgeschichte, in: Zeitschrift für historische Forschung 32 (2006), S. 387-415.

14 Vgl. Rebekka Habermas/Gerd Schwerhoff, Vorbemerkungen, in: Dies. (Hg.), Verbrechen im Blick, Frankfurt a. M./New York 2009, S. 9-18, hier S. 11.

15 Dorothee Rippmann/Katharina Simon-Muscheid/Christian Simon, Arbeit Liebe - Streit. Texte zur Geschichte des Geschlechterverhältnisses und des Alltags. 15. bis 18. Jahrhundert, Liestal 1996, S. 27-30.

16 Vgl. Rebekka Habermas, Rechts- und Kriminalitätsgeschichte revisited. Ein Plädoyer, in: Dies./Schwerhoff, Verbrechen im Blick (wie Anm. 14), S. 19-41.

17 „Geständnisse standen ebenso wie Klagen und Zeugenaussagen stets unter dem Imperativ des strategischen Anliegens, sich den Vertretern der Obrigkeit möglichst positiv darzustellen, die eigene Rolle bei verbotenen Tätigkeiten zu negieren oder wenigstens zu minimieren, sich und andere vor drohender Strafverfolgung zu schützen und mögliche Gegner in Verruf zu bringen." Gerd Schwerhoff, Historische Kriminalitätsforschung (Historische Einführungen 9), Frankfurt a. M./New York 2011, S. 67. 
eingegrenzt werden. Zum einen konzentriert sich die Untersuchung auf Fragen zur Person (Geschlecht, Alter etc.), die nur bedingt falsch beantwortet werden konnten, zum anderen auf Tätigkeitsangaben zu Arbeit, Zeit und Fleiß. Diese Aspekte standen meist nicht in einem direkten Bezug zum verhandelten Tatbestand, sondern flossen eher nebensächlich in die Aussagen ein und sind als ,unwillkürliche Überlieferung' zu betrachten. Im Unterschied $\mathrm{zu}$ Beobachtungen von Kriminalitätshistorikern wie Kienitz ${ }^{18}$ finden sich in den von uns erhobenen Daten, wie auch in Voths Untersuchung, nur geringfügige Hinweise auf strategische Aussagenverfälschungen, die auf die Berücksichtigung im Alltag wahrgenommener moralischer Restriktionen beziehungsweise sozialer Erwartungen zurückgehen. So nennen Zeugen und Beklagte in den untersuchten Verhörprotokollen häufig unerwünschte oder unerlaubte Tätigkeiten für die fraglichen Zeiten, wenn diese nicht im Zusammenhang mit der verhandelten Sache stehen; vermutlich um die eigene Vertrauenswürdigkeit zu beweisen. Strukturelle Auslassungen und Verzerrungen betreffen insofern offensichtlich nur den eigentlichen Prozessgegenstand, Handlungsmuster und Motive und somit Aspekte, die für die Frage der Zeitverwendung nicht relevant sind. Andererseits attestieren Fuchs und Schulze Zeugen allgemein eine relativ hohe Glaubwürdigkeit, ${ }^{19}$ aufgrund der abschreckenden Wirkung weltlicher und göttlicher Strafen im Fall von Falschaussagen (vor allem durch eine Vereidigung).

\section{b) Zuverlässigkeit der Verschriftlichung}

Als Zweites ist die Reliabilität der Überlieferung zu hinterfragen, d. h. ob die Mitschriften der Gerichtsschreiber das Gesagte zuverlässig wiedergeben und in welchem Umfang den Schreibern eine Filterfunktion zukam. ${ }^{20}$ In den Akten finden sich mehrheitlich summarische und typisierende Zusammenfassungen individueller Aussagen. Nur in Ausnahmefällen können das gesprochene Wort, Gefühlsäußerungen und Gesten nachvollzogen werden, denn vor allem in den summarischen Verhören kam es zu einer Umschreibung des gesprochenen Wortes in das Hochdeutsche (Kanzleisprache), zudem konnten die Aussagenden den geschriebenen Text nur in Einzelfällen überprüfen. Viele dieser Vorbehalte treffen jedoch auch auf andere ,Ego-Dokumente“ zu. „Bei einem Vergleich der Zuverlässigkeit schneiden die Gerichtsakten gar nicht so schlecht ab, weil der rechtliche Rahmen auch ein wichtiges Korrektiv für die Ermittlung des Wahrheitsgehaltes darstellen kann." 21 Andererseits dienten die Protokolle der Strafprozesse meist übergeordneten Instanzen als Entscheidungsgrundlage, sodass die

18 Vgl. Sabine Kienitz, Sexualität, Macht und Moral: Prostitution und Geschlechterbeziehungen Anfang des 19. Jahrhunderts in Württemberg. Ein Beitrag zur Mentalitätsgeschichte (Zeithorizonte 2), Berlin 1995, S. 67.

19 Fuchs/Schulze, Zeugenverhöre als historische Quellen (wie Anm. 8), S. 26-28.

$20 \mathrm{Zu}$ dieser Kontroverse vgl. Hagen Schulze, Mentalitätsgeschichte. Chancen und Grenzen eines Paradigmas der französischen Geschichtswissenschaft, in: Geschichte in Wissenschaft und Unterricht 36 (1985), S. 247-270; David Warren Sabean, Property, production, and family in Neckarhausen, 1700-1870 (Cambridge studies in social and cultural anthropology 73), Cambridge 1997, S. 76 f.; Silke GöTtsch, Weibliche Erfahrungen um Körperlichkeit und Sexualität. Ein Beispiel, in: Anita Chmielewski-Hagius (Hg.), Frauenalltag - Frauenforschung, Frankfurt a. M./Bern/New York/Paris 1988, S. 49-59, hier S. 49 f.; Klaus Graf, Das leckt die Kuh nicht ab. ,Zufällige Gedanken` zu Schriftlichkeit und Erinnerungskultur der Strafgerichtsbarkeit, in: Schwerhoff/Blauert, Kriminalitätsgeschichte (wie Anm. 10), S. 245-288; Carlo Ginzburg, Der Käse und die Würmer. Die Welt eines Müllers um 1600 (Taschenbücher Syndikat EVA 10), Frankfurt a. M. 1983, S. 14 f.

21 SChwerhoff, Historische Kriminalitätsforschung (wie Anm. 17), S. 68. 
Schreiber zur Genauigkeit verpflichtet waren und Flüchtigkeiten in der Niederschrift, bewusste oder unbewusste Sinnveränderungen durch den Schreiber unwahrscheinlich erscheinen. Auch die Verhöre selbst offenbaren mit ihren zahlreichen Redundanzen, Nichtigkeiten und Abschweifungen, dass eine Glättung nur selten erfolgt zu sein scheint. $^{22}$

\section{c) Vergessensrate}

Voths Kritiker machen bei den von ihm verwendeten Quellen eine weitere Form der Verfälschung beziehungsweise Ungenauigkeit aus, die in der relativ langen Zeit angelegt sei, die zwischen der Beobachtung eines Verbrechens oder eines Täters usw. durch den Zeugen und seinem ersten Verhör liegt. Dieser Zeitraum beträgt bei Voths Erhebungen rund 40 Tage, sodass Penelope J. Corfield eine entsprechend hohe Vergessensrate unterstellen kann. ${ }^{23}$ Auf Basis der von uns erfassten Daten ergab sich für die sächsischen Gerichte auf Ämter- und Kommunalebene ein deutlich kürzerer Zeitraum zwischen Verbrechen und Verhör von durchschnittlich 18 Tagen.

\begin{tabular}{lc}
\hline Abstand & Anteil in Prozent \\
\hline Kein zeitlicher Abstand & 18 \\
1 Tag & 46 \\
$2-7$ Tage & 15 \\
1-4 Wochen & 5 \\
Mehr als 1 Monat & 16 \\
\hline
\end{tabular}

Tab. 1: Zeitlicher Abstand zwischen Straftat und Verhör. Quelle: Eigene Berechnung.

Wie Tabelle 1 zeigt, fand in zwei Dritteln der Fälle ein erstes Verhör noch am Tag der Straftat beziehungsweise ein bis zwei Tage danach statt. Abweichend davon wurde ein geringer Teil (vor allem Kindsmorde) erst Wochen nach der Tat untersucht, sodass in diesem Zusammenhang durchaus ein langer Zeitraum zwischen Beobachtung und Aussage verging. ${ }^{24}$ Überwiegend kann jedoch eine relativ geringe Vergessensrate unterstellt werden, verstärkt durch die Tatsache, dass Verbrechen, Brände etc. außergewöhnliche Ereignisse darstellten, die den Alltag durchbrachen und vermutlich einen bleibenden Eindruck im Gedächtnis der Zeugen hinterließen.

\section{d) Repräsentativität der Zengen für die Studie}

Das vierte Problemfeld der externen Validität wird durch die Frage markiert, ob die Stichprobe der Personen, die bereit waren vor Gericht auszusagen beziehungsweise die zu Aussagen herangezogen wurden, repräsentativ für die damalige sächsische Gesellschaft ist. Voths Daten wurden auf verschiedenen Ebenen kritisiert, da die Anteile unterschiedlicher Bevölkerungsgruppen durch die Zeugen nicht charakteristisch abgebildet würden. So beschränken sich die von ihm ausgewerteten Zeugenverhöre zum einen ausschließlich auf Männer, zum anderen ist der Anteil ländlicher Bevölkerungsgruppen als zu gering bewertet worden, um Aussagen zum Arbeitsverhalten der Ge-

22 Ebd., S. 68.

23 Vgl. Penelope J. Corfield, Rezension von: Hans-Joachim Voth, Time and Work in England, 1750-1830, Oxford 2001, in: Business History Review 76 (2002), S. 188-192.

24 Bereinigt man die Berechnung um die 16 Prozent der Fälle, in denen mehr als ein Monat zwischen Tat und Verhör liegt, ergibt sich ein Durchschnittswert von 2,2 Tagen. 
samtbevölkerung treffen zu können. ${ }^{25}$ Diese letztgenannte Gefahr kann umgangen werden, da der sächsische Untersuchungsraum die Möglichkeit bietet, eine große Anzahl lokaler und landesherrlicher Gerichtsinstanzen zu berücksichtigen. Für das gesamte sächsische Territorium (Stadt und Land) liegen Quellen vor, sodass eine flächendeckende Erhebung möglich ist. Des Weiteren werden die Daten anhand der umfangreichen bevölkerungshistorischen Forschungsliteratur zum sächsischen Gebiet $^{26}$ und der zeitgenössischen publizierten Statistik ${ }^{27}$ reflektiert.

Momentan gestaltet sich die Verteilung der Zeugen folgendermaßen: In unserem Datensatz überwiegen Männer in der Rolle von Zeugen, doch können Frauen (mit geringen Einschränkungen) ebenfalls berücksichtigt werden, auch wenn ihr Anteil nicht der realen Verteilung entspricht (Abb. 1).

Bezüglich der Altersverteilung der Zeugen offenbart sich eine Schwierigkeit im Hinblick auf Kinder. Aussagen von unter 12-Jährigen waren nicht zulässig, sodass diese Gruppe fehlt. Unter Berücksichtigung von indirekten Angaben über Kinder und ihre Tätigkeiten zu bestimmten Zeiten durch andere Aussagende können jedoch auch begrenzt Angaben zur Kinderarbeit gemacht werden. Die für Arbeit und Fleiß relevante Personengruppe der 15- bis 60-Jährigen ist im Vergleich zur Gesamtbevölkerung überproportional vertreten (Abb. 2).

Möchte man die Berufsstruktur der sächsischen Bevölkerung mit einbeziehen, wird das Fehlen detaillierter zeitgenössischer Daten ersichtlich. Die Erhebung einer Bevölkerungsstatistik, die gleichzeitig die berufliche Tätigkeit berücksichtigt, setzte

25 Voth untersucht Aktenbestände aus zwei sehr unterschiedlichen Regionen - zum einen die Londoner Old Bailey Session Papers, zum anderen Akten aus sechs ländlich geprägten Bezirken in Nordengland (Northern Assizes). Die Londoner Prozesse vor dem Old Bailey, dem zentralen Strafgerichtshof seit 1748, sind präzise und detailliert, teilweise mit wörtlichen Zitaten der Beteiligten in den städtischen Zeitungen publiziert worden. Bei den Depositions dagegen bezieht er sich auf gekürzte Abschriften von Gerichtsprozessen, die von Friedensrichtern verhandelt wurden.

26 Vgl. Karlheinz BlaschKe, Bevölkerungsgeschichte von Sachsen bis zur industriellen Revolution, Weimar 1967; Francesco Cinnirella, On the Road to Industrialization: Nutritional Status in Saxony, 1690-1850, in: Cliometrica 3 (2008), S. 229-257; UlF CHristian EwErT, Beeinträchtigungen und Beschädigungen des Körpers in Sachsen im 18. und 19. Jahrhundert. Möglichkeiten der Auswertung von Militärakten, in: Cordula Nolte (Hg.), Phänomene der ,Behinderung' im Alltag. Bausteine zu einer Disability History der Vormoderne (Studien und Texte zur Geistes- und Sozialgeschichte des Mittelalters 8), Affalterbach 2013, S. 273-292; Ulf Christian Ewert, Die ,Kleinen Leute $^{6}$ in Sachsens Frühindustrialisierung. Zum sinkenden Lebensstandard einer wachsenden Bevölkerung in der ersten Hälfte des 19. Jahrhunderts, in: Jahrbuch für Regionalgeschichte 25 (2007), S. 45-70; Michael Kopsidis/Ulrich Pfister, Agricultural development during early industrialization in a low-wage economy: Saxony, c. 1790-1830, in: European Historical Economics Society Working Papers in Economic History 39 (2013), http://ehes.org/EHES_No39.pdf [Zugriff am 27. Juni 2014]; UwE SCHIRMER, Der Bevölkerungsgang in Sachsen zwischen 1743 und 1815, in: Vierteljahrschrift für Sozial- und Wirtschaftsgeschichte 83 (1996), S. 25-58; VolKMAR WeIss, Bevölkerung und soziale Mobilität. Sachsen 1550-1880, Berlin 1993.

27 Vgl. Friedrich Anton von Heynitz, Tabellen über die Staatswissenschaft eines europäischen Staates vierter Größe, [Leipzig] 1786; FrIedrich GotTlob LeONHARdi, Erdbeschreibung der Churfürstlich- und Herzoglich-Sächsischen Lande, Bd. 1, Leipzig 1802; Karl-Heinrich Pölitz, Geschichte, Statistik und Erdbeschreibung des Königreichs Sachsen, Teil 2, Leipzig 1810; Robert WuttKe, Sächsische Volkskunde, Leipzig 1903. 


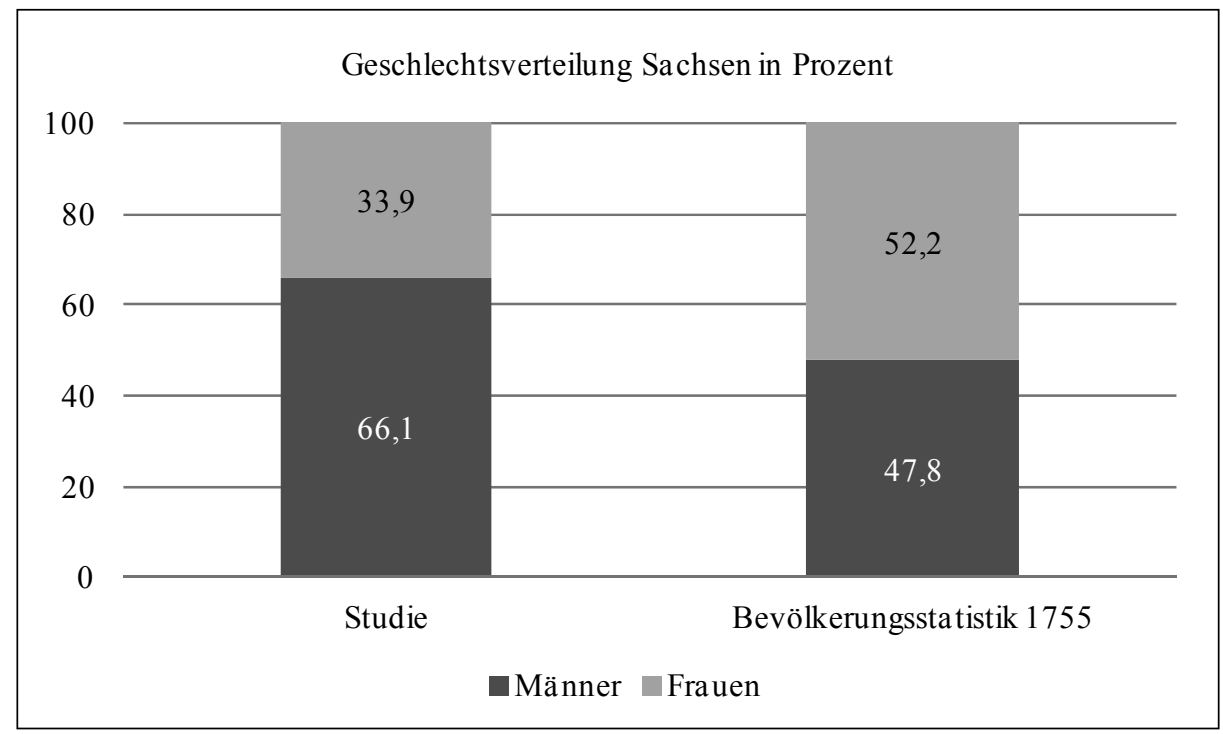

Abb. 1: Verteilung der Zeugen nach Geschlecht in Vorstudie und Bevölkerungsstatistik 1755. Quelle: Eigene Berechnung und ROBERT WUTTKE, Sächsische Volkskunde, Leipzig 1903, S. 193.

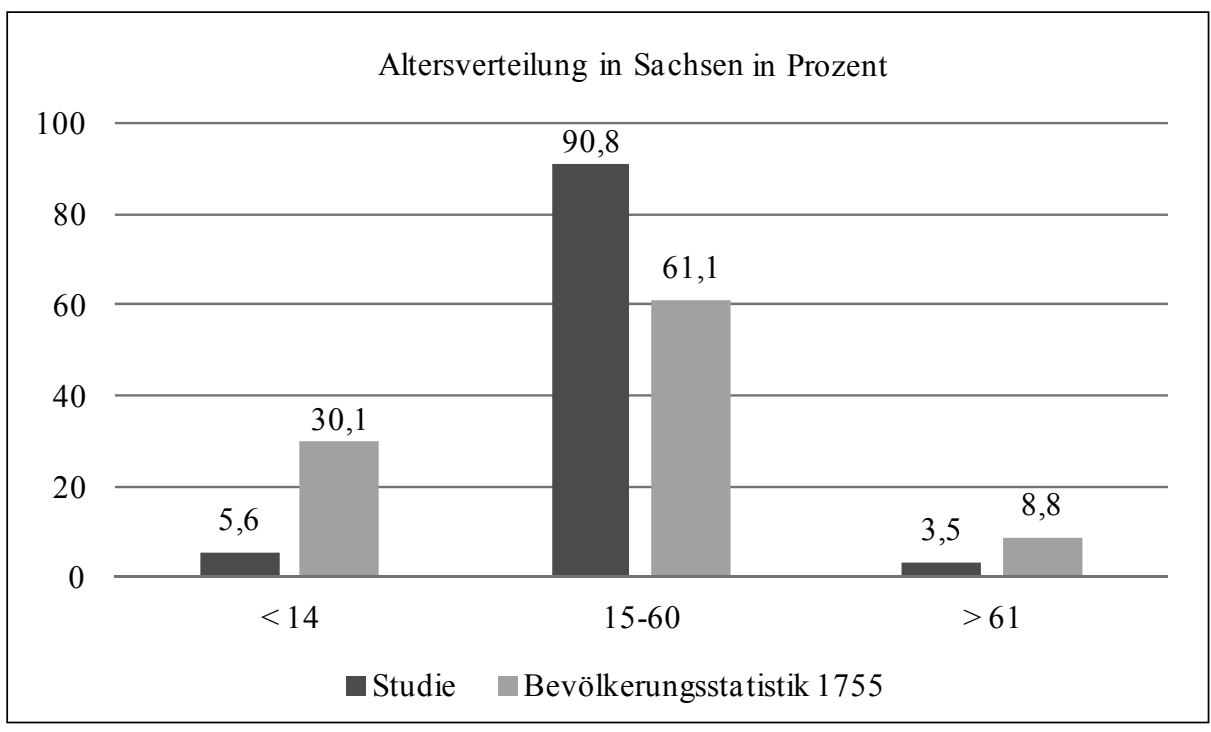

Abb. 2: Altersstruktur der Zengen. Quelle: Eigene Berechnung und ROBERT WUTTKE, Sächsische Volkskunde, Leipzig 1903.

erst um 1800 ein. Zieht man diese lückenhaften historischen Erhebungen und ihre nicht immer nachvollziehbaren Differenzierungen dennoch als Vergleich heran, offenbart sich für die Studie eine Dominanz von Gesinde und Tagelöhnern. Die Zuordnung in diese Gruppe ist jedoch nicht immer zweifelsfrei möglich. Daneben finden sich momentan weniger Handwerker, dafür jedoch mehr im Handel Tätige als in der zeit- 
genössischen Statistik. Auch die Forschungsliteratur liefert kaum belastbare Angaben zur beruflichen Differenzierung der sächsischen Gesamtbevölkerung. So analysiert Weiß beispielsweise die soziale und räumliche Mobilität und berücksichtigt in diesem Zusammenhang auch den Beruf der Betreffenden, doch basiert seine Studie auf einer Gegenüberstellung der Berufe des Vaters und des Sohnes, um mithilfe dieser Spiegelung den sozialen Auf- oder Abstieg dokumentieren zu können. Für eine Strukturierung der Gesamtbevölkerung reichen diese vereinzelten Daten hingegen nicht aus.

Ebenfalls problematisch gestaltet es sich, eine noch detailliertere Differenzierung nach Beruf und Geschlecht vorzunehmen. In vielen Verhören bezeichneten sich Frauen als, Witwe' oder ,Soldatenfrau', was zunächst keine Rückschlüsse auf ihre berufliche Tätigkeit zulässt, eine solche aber auch nicht grundlegend ausschließt. Bei Handwerkerfrauen kann und muss beispielsweise davon ausgegangen werden, dass sie sich ebenfalls im Betrieb betätigten, ohne dies explizit als ihren Beruf anzusehen und anzugeben. Um der damaligen Lebenswirklichkeit gerecht $\mathrm{zu}$ werden, erscheint es darum angemessener, Tätigkeitsbereiche (Handwerk, Handel, Landwirtschaft etc.) abzustecken und als Analysekategorien zu verwenden, anstatt mit dem Begriff ,Beruf ${ }^{\star}$ und einer daraus abgeleiteten Systematik zu arbeiten. Eine Minimaldifferenzierung nach ländlicher und nicht-ländlicher Betätigung ermöglicht bereits jetzt eine gewisse Repräsentativität. In Abbildung 3 wird deutlich, dass die Daten der Studie denselben Trend des Rückgangs von in der Landwirtschaft Beschäftigten für Sachsen aufweisen, wie dies auch von Kopsidis/Pfister konstatiert wird.

Ein grundlegendes Quellenproblem betrifft die Selektion der Überlieferung: Zum einen vermuten Historiker bei verschiedenen Delikten hohe Dunkelziffern, zum anderen gingen Akten oder ganze Archivbestände aufgrund äußerer Umstände verloren oder wurden bewusst vernichtet. Beide Schwierigkeiten tangieren jedoch nicht die Ermittlung historischer Zeitbudgets.

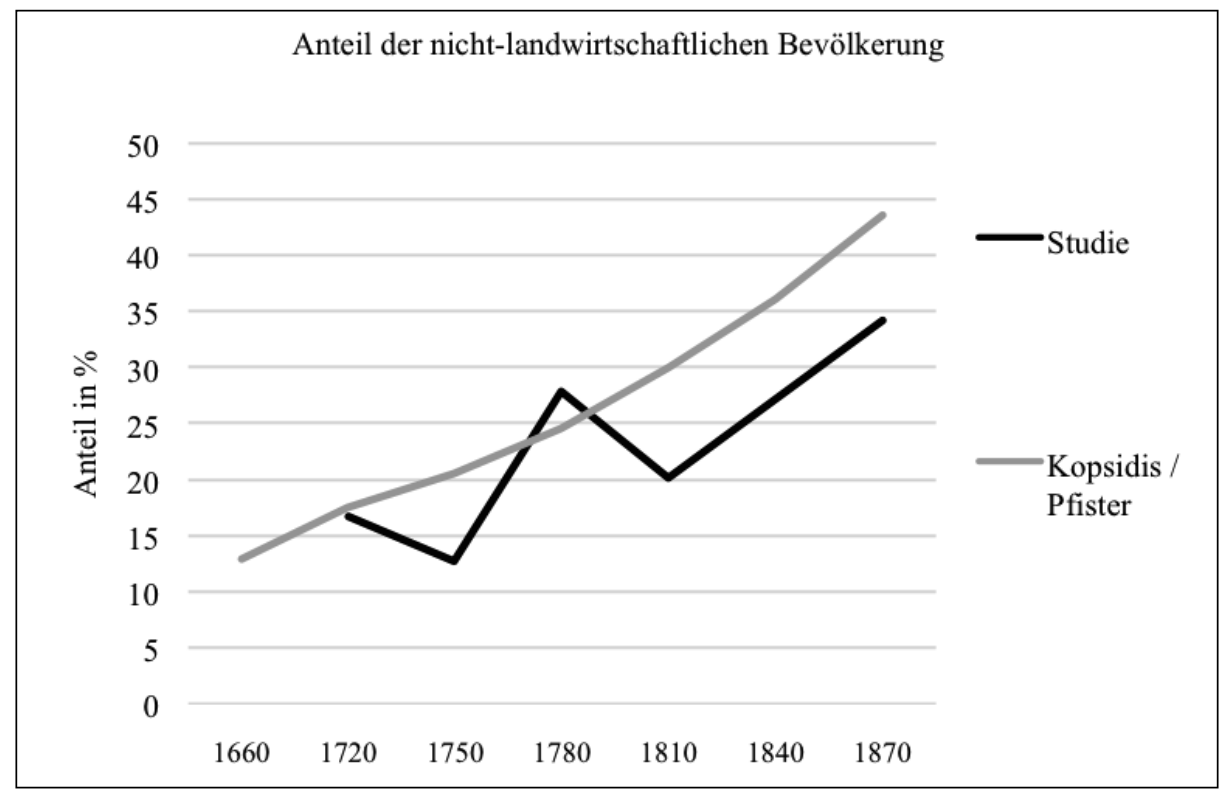

Abb. 3: Anteil der nicht-landwirtschaftlichen Bevölkerung. Quelle: Eigene Berechnung und KOPSIDIS/ PFISTER, Agricultural development (wie Anm. 26), S. 5. 


\section{Anzabl der zu erfassenden Verbördaten unter Berücksichtigung der anzurendenden Methoden}

Die Ermittlung historischer Zeitbudgets aus Prozessakten zielt darauf, Arbeitszeiten im 17., 18. und 19. Jahrhundert abzuschätzen und zu vergleichen. Die Konfidenz (Vertrauenswürdigkeit), mit der sich auf Basis quantitativer Daten Aussagen beispielsweise zur täglichen Arbeitszeit treffen lassen können, hängt unmittelbar von der Größe des Stichprobenumfanges ab. Er kann unter Berücksichtigung des in der Auswertung anzuwendenden Verfahrens bestimmt werden. Im Folgenden werden drei Möglichkeiten der statistischen Ermittlung historischer Zeitbudgets verglichen und u. a. anhand der Abschätzung ihres jeweils nötigen Stichprobenumfangs auf ihre Anwendungsmöglichkeit im Projekt hin überprüft. Untersucht wird die Frage nach der täglichen Arbeitszeit.

\section{Direkte Angaben der mittleren Arbeitsdauer}

Bei diesem Verfahren werden die Mittelwerte von Arbeitszeiten in verschiedenen Zeitintervallen verglichen. Die Angabe einer täglichen Arbeitsdauer und Pausenzeit innerhalb der Aussagen ist jedoch äußerst selten. Beim derzeitigen Stand der Studie gibt es lediglich zwei Zeugen, die mehr als fünf Angaben zu ihren Tätigkeiten zu bestimmten Zeiten eines gewissen Tages machen. Bei dieser Datenlage kann keine Abschätzung des Stichprobenumfanges erfolgen. Dieses Verfahren ist im Projekt somit voraussichtlich nicht anwendbar.

\section{Direkte Angaben zu Anfang und Ende der Arbeit}

Diese Methode, bei der die Mittelwerte von Aufstehzeit, Arbeitsbeginn, Arbeitsende und Zubettgehzeit in verschiedenen Zeitintervallen verglichen werden, nutzt Voth zur Berechnung der Tagesarbeitszeit. In einem Aufsatz von 2001 vergleicht er Angaben zur Aufstehzeit von zwei Zeitintervallen des 18. beziehungsweise 19. Jahrhunderts. ${ }^{28}$ Voth kann sich jeweils auf ca. 40 verwertbare Datenpunkte aus Zeugenaussagen stützen. Auf Basis dieses Stichprobenumfanges ist es nicht möglich, vergleichende Aussagen zu einer Veränderung der Aufstehzeit zu treffen, da sich die Konfidenzintervalle überlagern und sich die mittleren Zeiten damit nicht signifikant unterscheiden. Eine Aussage zur Veränderung wäre erst möglich, wenn Voth seine Stichprobenumfänge in beiden Zeiträumen auf 200 Datenpunkte vergrößern würde. Unter den bereits erhobenen Daten der Studie gibt es (bei 161 in dieser Frage nutzbaren Datenpunkten) im gesamten Untersuchungszeitraum sieben Personen, die ihre Aufstehzeit angeben. Eine Richtzahl von 200 Datenpunkten pro zu vergleichendem Zeitraum (wenn man eine ähnliche Streuung der Daten, wie Voth sie vorfand, annimmt) kann nach Hochrechnung der bereits erhobenen Daten im Rahmen dieses Projektes vermutlich nicht erreicht werden. Das Verfahren des Vergleichs der Angaben zu mittlerem Anfang und mittlerem Ende der Arbeit kann voraussichtlich nicht angewendet werden. Ein Nachteil dieser Methode ist zudem, dass lediglich über eine zeitliche Verschiebung von Arbeitsbeginn und -ende Aussagen getroffen werden können. Die eventuell freien Stunden dazwischen (Essen, Kirchbesuch, Mittagsschlaf etc.) werden nicht erfasst. Daneben ist die Effizienz der Datenverwertung sehr gering, da nur die Angaben zur Aufsteh- und Zubettgehzeit, nicht aber alle anderen Angaben zu Arbeit beziehungsweise Nicht-Arbeit genutzt werden können.

28 Hans-Jоаснім Voth, The Longest Years: New Estimates of Labor Input in England, 1760-1830, in: The Journal of Economic History 61 (2001), S. 1065-1082. 


\section{Zeitabschnittsweise Bestimmung des Anteils von Arbeit an den genannten} Tätigkeiten

Auch diese Methode nutzt Voth in seiner Studie, um zu bestimmen, an welchen Wochentagen gearbeitet oder nicht gearbeitet wurde. Das Verfahren kann jedoch auch zur Berechnung der täglichen Arbeitszeit genutzt werden. Für eine möglichst realitätsnahe Ermittlung der nötigen Stichprobengröße konnten die bisherigen Erkenntnisse der Studie genutzt werden. Der Tagesverlauf wurde anhand der Zusatzangaben der Zeugen, die oft über die Nennung der reinen Tageszeit hinaus erfolgten, in unten stehende Tageszeitintervalle eingeteilt (früh: 5-8 Uhr, vormittags: 8-12 Uhr usw.). Daneben erschien es sinnvoll, auf Basis der noch beschränkten Datengrundlage (hier 161 verwertbare Datenpunkte) den Anteil der genannten Tätigkeiten nur vergleichend für vor 1800 und nach 1800 zu ermitteln. Es wurde zunächst bestimmt, welchen Anteil als Arbeit klassifizierte Tätigkeiten in dem jeweiligen Intervall haben. Dieser Wert bildet einen Schätzer für den binominal verteilten Anteil an Arbeitszeit in der Gesamtbevölkerung. Als Beurteilungsgröße der Genauigkeit dieser Schätzwerte dient das Konfidenzintervall. Es wurde unter Annahme einer Normalverteilung der Abweichung zwischen Schätzwert und Erwartungswert der Grundgesamtheit ermittelt. Eine ausreichende Genauigkeit wird normativ als Konfidenzintervall eines bestimmten Abstandes definiert, beispielsweise 5 oder 10 Prozent. Damit konnte die notwendige Stichprobengröße unter Berücksichtigung des Schätzers für den Erwartungswert berechnet werden. Die folgenden Tabellen geben eine Übersicht über die Stichprobengrößen in Abhängigkeit vom gewünschten Konfidenzintervall vergleichend für vor 1800 und nach 1800:

\begin{tabular}{cccccc}
\hline Zeitspanne & $\begin{array}{c}\text { Stich- } \\
\text { proben- } \\
\text { größe(n) im } \\
\text { Datenset }\end{array}$ & $\begin{array}{c}\text { Anteil } \\
\text { Arbeit in } \\
\text { Prozent }\end{array}$ & $\begin{array}{c}\text { einseitiges } \\
\text { Konfi- } \\
\text { denzinter- } \\
\text { vall (KI) }\end{array}$ & $\begin{array}{c}\text { n bei KI }= \\
15\end{array}$ & $\begin{array}{c}\text { n bei KI }= \\
10\end{array}$ \\
\hline 05.00-08.00 Uhr & 3 & 67 & 53 & 38 & 85 \\
08.00-12.00 Uhr & 21 & 62 & 21 & 40 & 91 \\
$12.00-13.30 \mathrm{Uhr}$ & 11 & 18 & 23 & 25 & 57 \\
$13.30-17.00 \mathrm{Uhr}$ & 2 & - & - & - & - \\
$17.00-23.00 \mathrm{Uhr}$ & 7 & 14 & 26 & 21 & 47 \\
$23.00-05.00 \mathrm{Uhr}$ & 7 & - & - & - & - \\
\hline Gesamt & 51 & 35 & 13 & 124 & 280 \\
\hline
\end{tabular}

Tab. 2: Erforderliche Stichprobengrößen in Abhängigkeit vom gewünschten Konfidenzintervall 16001800. 


\begin{tabular}{cccccc}
\hline Zeitspanne & $\begin{array}{c}\text { Stich- } \\
\text { proben- } \\
\text { größe(n) im } \\
\text { Datenset }\end{array}$ & $\begin{array}{c}\text { Anteil } \\
\text { Arbeit in } \\
\text { Prozent }\end{array}$ & $\begin{array}{c}\text { einseitiges } \\
\text { Konfi- } \\
\text { denzinter- } \\
\text { vall (KI) }\end{array}$ & $\begin{array}{c}\text { n bei KI }= \\
15\end{array}$ & $\mathrm{n}$ bei KI $=$ \\
10 & \\
\hline 05.00-08.00 Uhr & 6 & 50 & 40 & 43 & 96 \\
08.00-12.00 Uhr & 21 & 38 & 21 & 40 & 91 \\
$12.00-13.30 \mathrm{Uhr}$ & 7 & 43 & 37 & 42 & 94 \\
$13.30-17.00 \mathrm{Uhr}$ & 6 & 33 & 38 & 38 & 85 \\
17.00-23.00 Uhr & 41 & 24 & 13 & 31 & 71 \\
23.00-05.00 Uhr & 24 & 21 & 16 & 28 & 63 \\
\hline Gesamt & 105 & 30 & 9 & 222 & 500 \\
\hline
\end{tabular}

Tab. 3: Stichprobengrößen in Abhängigkeit vom gewünschten Konfidenzintervall 1800-1900.

Die vorgestellte Abschätzung erfolgte teilweise auf Grundlage sehr kleiner Teilstichproben (Intervalle 05.00-08.00 Uhr und 13.30-17.00 Uhr vor 1800), weshalb die daraus ermittelten Schätzwerte kaum aussagekräftig sind. Um eine robuste Abschätzung für die notwendige Stichprobengröße zu erhalten, wurden darum der niedrigste und der höchste Wert der für die einzelnen Zeitspannen ermittelten Daten angenommen (KI 15: $21<\mathrm{n}>43$ ). Folgende Stichprobengrößen in Abhängigkeit von den zu betrachtenden Zeitintervallen pro Tag und den Zeitschritten, in die der Gesamt-Untersuchungszeitraum eingeteilt werden soll, ergeben sich daraus:

\begin{tabular}{|c|c|c|c|c|}
\hline \multirow{2}{*}{$\begin{array}{l}\text { Zeitintervalle } \\
\text { pro Tag }\end{array}$} & \multicolumn{4}{|c|}{ Zeitschritte Gesamtzeitraum (1600-1900) in Jahren } \\
\hline & 10 & 25 & 50 & 100 \\
\hline 1 & $630-1290$ & $252-516$ & $126-258$ & $63<n>129$ \\
\hline 2 & $1260-2580$ & 504-1032 & $252-516$ & $126-258$ \\
\hline 4 & $2520-5160$ & $1008-2064$ & $504-1032$ & $252-516$ \\
\hline 6 & $3780-7740$ & $1512-3096$ & $756-1548$ & $378-774$ \\
\hline 8 & $5040-10320$ & $2016-4128$ & $1008-2064$ & $504-1032$ \\
\hline 12 & $7560-15480$ & $3024-6192$ & $1512-3096$ & $756-1548$ \\
\hline 24 & $15120-30960$ & $6048-12384$ & $3024-6192$ & $1512-3096$ \\
\hline
\end{tabular}

Tab. 4: Gesamt-Stichprobengrößen in Abbängigkeit von den zu betrachtenden Zeitintervallen.

Die ermittelten Stichprobengrößen für die Frage nach der täglichen Arbeitszeit stellen den Grundfaktor dar. Je nach Fragestellung erhöht sich die jeweilige Stichprobengröße in Abhängigkeit zu erwartender Faktoren für die Teilmengen. So ergeben sich bei einer Unterscheidung nach dem Geschlecht zwei Teilmengen, nach Wochentagen sieben usw. Somit liegt die Stichprobengröße bei der Frage danach, ob im 17., 18. oder 19. Jahrhundert Veränderungen der sonntäglichen Arbeitszeit stattfanden, zwischen 2646 und 5418 Datenpunkten $([378<n>774] * 7=[2646<n>5418])$. 


\section{Datenlage und Datenerfassung}

Für die explorative Datenerhebung wurden folgende Schritte unternommen: 1. eine stichprobenartige Untersuchung von Straf- und Zivilprozessen in den Aktenbeständen des Hauptstaatsarchivs Dresden und des Staatsarchivs Leipzig auf das Vorhandensein von Datenpunkten hin, 2. eine umfassende Analyse der relevanten Findbücher in den Archiven Dresden und Leipzig verbunden mit einer Kategorisierung der unterschiedlichen Delikte, 3. eine stichprobenweise detaillierte Sichtung einzelner Akten unter Berücksichtigung der gebildeten Kategorien, eine Erhebung von Datenpunkten aus den unterschiedlichen Gerichtsakten auf Grundlage einer relationalen Datenbank und eine Analyse der Kategorien hinsichtlich ihrer Ergiebigkeit sowie 4. eine Hochrechnung der zu erwartenden Datenpunkte in den Archiven Dresden und Leipzig. Danach konnten 5. die Probleme und Fehler bei der Datenerhebung betrachtet und 6. erste Auswertungen des Quellenertrages vorgenommen werden.

\section{Betrachtungen von Straf- und Zivilprozessen auf das Vorhandensein von Datenpunkten hin}

Aus dem geplanten Untersuchungszeitraum (1600-1900) wurden unterschiedliche sächsische Straf- und Zivilprozessakten gesichtet. Bei ihrer Betrachtung stand die Frage im Vordergrund, ob die entsprechenden Unterlagen die benötigten Verhörprotokolle beziehungsweise andere Formen aufgezeichneter Verhöre oder Befragungen beinhalten und ob diese verwendbare Zeit- und Tätigkeitsangaben aufweisen. Nach der ersten Sichtung ergab sich, dass die Mehrheit aller Strafprozesse über unterschiedlich umfangreiche Verhörprotokolle verfügt und diese auch detaillierte und verknüpfte Zeit- und Tätigkeitsangaben bieten, während die Zivilprozesse in den meisten Fällen zwar ebenfalls komplexe Verhöre und Befragungen beinhalten, diese sich jedoch auf Streitigkeiten konzentrieren, denen keine konkreten Zeit- und Tätigkeitsangaben zuzuordnen sind. In den meisten Fällen (Wegestreit, Grenzkonflikt, Erbschaften oder Konzessionen) beziehen sich die protokollierten Angaben in Zivilprozessakten auf das Gewohnheitsrecht und werden nicht mit konkreten Tätigkeiten zu einem bestimmten Zeitpunkt in Beziehung gesetzt. Aufgrund dieser Feststellung wurden die Zivilprozessakten, trotz ihres großen Anteils an den überlieferten Gerichtsunterlagen, für die vorliegende Studie vernachlässigt.

\section{Analyse der Findbücher und Kategorisierung der Straftatbestände}

Um einen Eindruck von den Möglichkeiten und Grenzen einer auf historischen Kriminalitätsakten beruhenden Analyse zu erhalten, wurden zunächst die in Dresden und Leipzig vorhandenen Strafprozessakten systematisch erfasst und kategorisiert. ${ }^{29} \mathrm{Im}$ vorliegenden Fall betrifft die Kategorienbildung hauptsächlich die Erstellung von Deliktkategorien. Diese sind auf der einen Seite zwingend erforderlich, um die Ergiebigkeit der einzelnen Straftatbestände abschätzen zu können und diejenigen auszu-

29 Für den Standort Chemnitz des Sächsischen Staatsarchivs und das Staatsfilialarchiv Bautzen wurden noch keine detaillierten Sichtungen vorgenommen, nach ersten Schätzungen gestaltet sich die Aktenlage dort jedoch ähnlich gut wie in Leipzig und Dresden. Im Staatsarchiv Chemnitz wurden nach einer ersten Bestandsaufnahme 30 Ämter, 297 Grundherrschaften, 8 Stadtgerichte, 37 Landesgerichte und 38 Amtsgerichte ausgemacht. Im Staatsfilialarchiv Bautzen finden sich 40 Amter, 304 Gutsherrschaften, 24 städtische Gerichte und 16 Königliche Gerichte. 
wählen, die eine höchstmögliche Menge an Datenpunkten versprechen, auf der anderen Seite problematisch, da sich normative Unschärfen nicht immer vermeiden lassen. Um eine Vergleichbarkeit zu ermöglichen, dürfen die Deliktkategorien einerseits nicht zu klein angelegt sein, andererseits stellt sich in diesem Zusammenhang häufig die Frage, welche Delikte in welche Großkategorien zu subsumieren sind. Gehört beispielsweise der Kindsmord zur Kategorie ,Mord, Totschlag' oder stellt er aufgrund seiner strafrechtlichen Behandlung einen Sondertatbestand dar? Unter Berücksichtigung dieser methodischen Probleme konnten zwölf Kategorien angelegt werden. Zu differenzieren sind dabei, aufgrund der behördlichen Zuständigkeiten, die verschiedenen Verwaltungs- und Gerichtsebenen in kommunale Ebene ${ }^{30}$, Ämter ${ }^{31}$ und Landesbeziehungsweise Reichsebene ${ }^{32}$. Die teilweise deutlich unterschiedliche Verteilung der Delikte auf diese drei Ebenen ist der jeweiligen Zuständigkeit geschuldet. Tabelle 5 zeigt eine Aufstellung der Bestände in den Archiven Dresden und Leipzig. Sie berücksichtigt noch nicht, ob in den unterschiedlichen Akten die für die Studie relevanten Verhörprotokolle enthalten sind. Diese Information kann nicht auf der Ebene der Bestandsübersicht auf Basis von Findbüchern gewonnen werden, da in ihnen keine inhaltliche Aufstellung der einzelnen Akten verzeichnet ist. $\mathrm{Zu}$ beantworten ist diese Frage somit nur durch den Blick in jede einzelne Akte.

\begin{tabular}{|c|c|c|c|c|c|}
\hline Delikt & $\begin{array}{c}\text { Kommunale } \\
\text { Ebene } \\
\text { Dresden }\end{array}$ & $\begin{array}{c}\text { Ämter } \\
\text { Dresden }\end{array}$ & $\begin{array}{c}\text { Kommunale } \\
\text { Ebene } \\
\text { Leipzig }\end{array}$ & $\begin{array}{l}\text { Ämter } \\
\text { Leipzig }\end{array}$ & $\begin{array}{l}\text { Landes- } \\
\text { und } \\
\text { Reichs- } \\
\text { ebene } \\
\text { Leipzig/ } \\
\text { Dresden }\end{array}$ \\
\hline $\begin{array}{l}\text { Aufwiegelung, } \\
\text { Friedensstörung, } \\
\text { Revolution }\end{array}$ & 221 & 234 & 42 & 230 & 166 \\
\hline Beleidigung & 95 & 48 & 113 & 40 & 150 \\
\hline $\begin{array}{l}\text { Betrug, } \\
\text { Glücksspiel, } \\
\text { Falschmünzerei }\end{array}$ & 96 & 169 & 23 & 21 & 48 \\
\hline $\begin{array}{l}\text { Brand, } \\
\text { Brandstiftung }\end{array}$ & 140 & 14 & 47 & 14 & 51 \\
\hline $\begin{array}{l}\text { Diebstahl, } \\
\text { Einbruch, Raub, } \\
\text { Überfall }\end{array}$ & 183 & 243 & 215 & 67 & 398 \\
\hline Konzession & 21 & 25 & 12 & 0 & 6 \\
\hline $\begin{array}{l}\text { Körperverletzung, } \\
\text { fahrlässige Tötung, }\end{array}$ & 72 & 42 & 87 & 22 & 82 \\
\hline
\end{tabular}

30 Diese betrifft in Dresden 8 Stadtgerichte und 83 Grundherrschaften, in Leipzig 13 Stadtgerichte und ebenfalls 83 Grundherrschaften.

31 Das Hauptstaatsarchiv Dresden weist 16 Ämter, das Staatsarchiv Leipzig 18 Ämter auf.

$32 \mathrm{Zu}$ dieser gehören in Dresden der Geheime Rat, das Appellationsgericht und das Reichskammergericht, in Leipzig ebenfalls das Appellationsgericht, 24 Gerichtsämter und 16 Königliche Gerichte. 


\begin{tabular}{lccccc}
$\begin{array}{l}\text { Leichenfund, } \\
\text { Selbstmord, } \\
\text { Todesfall }\end{array}$ & 2 & 8 & 42 & 31 & 5 \\
$\begin{array}{l}\text { Mord, Kindsmord, } \\
\text { Totschlag }\end{array}$ & 115 & 87 & 23 & 23 & 177 \\
$\begin{array}{l}\text { Presse, Zensur } \\
\text { Sittlichkeit und } \\
\text { ungebührliches }\end{array}$ & 50 & 19 & 3 & 13 & 111 \\
$\begin{array}{l}\text { Verhalten } \\
\text { Sonstiges }\end{array}$ & 91 & 52 & 113 & 36 & 126 \\
\hline $\begin{array}{l}\text { Summe } \\
\text { Gesamtbestand }\end{array}$ & 1235 & 1005 & 885 & 522 & 1445 \\
\hline
\end{tabular}

Tab. 5: Strafprozessakten nach Gerichtsebene und Deliktkategorie.

3. Sichtung einzelner Akten, Erhebung von Datenpunkten und Analyse ihrer Ergiebigkeit

Die Stichprobenerhebung zielte darauf, Aussagen zur Ergiebigkeit unterschiedlicher Deliktkategorien machen zu können. ${ }^{33}$ Sowohl für die Sichtung als auch für den generellen Umgang mit den Quellen entscheidend ist die Definition eines für die Studie relevanten Datenpunkts. Ein Datenpunkt benötigt zum einen die Erfassung und Erhebung sozialgeschichtlicher Daten zur Charakterisierung der beteiligten Personen. Diese betreffen neben Namen, Alter, Geschlecht und Beruf möglichst auch Wohnort und Familienstand. Im Einzelfall könnten die aus den Akten gewonnenen Informationen durch die Auswertung weiterer Quellentypen ergänzt werden, so z. B. durch Steuerlisten, Personenverzeichnisse oder Kirchenbücher. Neben diesen Angaben sind zur Erfassung historischer Zeitbudgets Informationen zu Tätigkeit(en) und Zeitpunkt(en) nötig. Während die personenbezogenen Daten sich meist in der Kopfzeile des summarischen Verhörs beziehungsweise in den ersten Fragen des artikulierten Verhörs finden, müssen Zeit- und Tätigkeitsangaben aus den teilweise sehr umfangreichen Aussagen extrahiert werden.

Zur Verdeutlichung der Datenpunkt-Generierung soll folgendes Beispiel dienen: In einem Kindsmordprozess, welcher 1716 vor dem Dresdener Appellationsgericht verhandelt wurde, ${ }^{34}$ sagt die Zeugin Catharina Meschkerin aus, dass sie sich Sonnabend, den 19. Juni 1715, nach 7 Uhr in der Früh im Haus einer Beklagten aufgehalten habe und mit der Wäsche beschäftigt gewesen sei. An späterer Stelle erläutert sie, dass sie mittags des gleichen Tages zur Beichte gegangen sei. Ihr Alter gibt sie mit ungefähr 50 Jahren an. Sie ist als Hilfe im Haus der angeklagten Wirtin tätig. Der erste relevante

33 Vernachlässigt wurden in diesem ersten Schritt die Kategorien Amtsbeschwerde, Aufwiegelung/Friedensstörung/Revolution, Beleidigung, Konzession, Presse und Zensur und summarische Zeugenanhörungen. Es kann jedoch begründet davon ausgegangen werden, dass diese Kategorien weniger Datenpunkte aufweisen.

34 Für das Folgende vgl. Untersuchungsakten. Anna Catharina verw. gewes. Wolff, verehl. Müller in Großröhrsdorf bei Pirna und Ehemann Michael Müller wegen Kindesmord, 1715-1720, Sächsisches Staatsarchiv - Hauptstaatsarchiv Dresden, Appellationsgericht 10084, Nr. 2537, fol. 128r/129v. 
Datenpunkt für die spätere Analyse, der sich aus dieser Aussage generiert, lautet: Catharina Meschkerin, weiblich, ca. 50 Jahre, Hausgehilfin, 29. Juni 1715, 7 Uhr Wäsche machen, der zweite relevante Datenpunkt: Catharina Meschkerin, weiblich, ca. 50 Jahre, Hausgehilfin, 29. Juni 1715, mittags, Beichte.

Als ,Mindestanforderung ' an einen Datenpunkt bedarf es einer Zeitangabe, diese kann sowohl sehr genau (7 Uhr) als auch vage (gegen Mittag) formuliert sein, und einer dazugehörigen Tätigkeit einer oder auch mehrerer Personen. Die Angaben zu den betreffenden Personen sind ebenfalls sehr variabel. Als eine grundlegende Information in diesem Zusammenhang muss das Geschlecht angesehen werden, während Name, Alter und Beruf meist nicht aus allen Aussagen gewonnen werden können. Aus diesem Grund ergeben sich bedeutende qualitative Unterschiede innerhalb der erhobenen Datenpunkte. Dies hat wiederum Rückwirkungen auf die Anzahl der aus einer Akte zu gewinnenden Datenpunkte. Abhängig davon, wie streng die angelegten Maßstäbe sind, kann der Datenpunkt-Ertrag einer Akte stark schwanken, was sich auch auf die Dauer der Datenerhebung auswirkt. Als ,harte Datenpunkte' sollen im Folgenden Datenpunkte bezeichnet werden, die alle relevanten Angaben und Informationen enthalten, während ,weiche Datenpunkte verschiedene Informationslücken aufweisen, grundlegend aber bei entsprechenden Fragestellungen in der Studie verwendbar bleiben. Das Verhältnis von ,harten' zu ,weichen Datenpunkten' liegt bei 1:5,6.

Im Rahmen der ersten Erhebung historischer Zeitbudgets konnten 274 ,harte Datenpunkte herausgearbeitet werden, die den Zeitraum 1680 bis 1870 umfassen. Es wurden Aussagen sowohl von Beschuldigten als auch von Zeugen aufgenommen. In ihnen können verschiedene ,Aussagevarianten' voneinander unterschieden werden. Aus jeder eindeutigen Zuordnung von Tätigkeit, Zeit und mindestens einer Person wurde ein Datenpunkt gebildet, vorausgesetzt dass für die betreffenden Personen auch alle anderen zwingend erforderlichen Informationen vorlagen.

Nach der quellenbasierten Aufnahme der Aussagen erfolgte eine Codierung sowohl der Zeitangaben als auch der verschieden ausführlich be- und umschriebenen Tätigkeiten. Die Angaben zur zeitlichen Verortung finden sich in einem breiten Spektrum und reichen von Umschreibungen (drei Stunden vor Aufgang der Sonne) über Angaben anhand des Kirchenjahrs (am Grünen Donnerstag) oder einer vagen Erklärung der Tageszeit (nach dem Mittagessen, am Abend) bis hin zu genauen Uhrzeiten. ${ }^{35}$ Für jede dieser unterschiedlichen Angaben mussten ein Wochentag und ein Datum ermittelt werden, was anhand des Verhördatums und verschiedener Umrechnungstabellen (z. B. Grotefend) ${ }^{36}$ möglich ist. Ein weiterer Schritt bestand in der Beantwortung der Frage, ob der betreffende Tag ein religiöser Feiertag war. Im Hinblick auf die Tätigkeiten erfolgte ebenfalls eine Codierung, um eine Analyse und eine Vergleichbarkeit zu ermöglichen. Grundlegend lassen sich in dieser Hinsicht zwei große Kategorien (Arbeit und Nichtarbeit) bilden, die sich beliebig weiter differenzieren lassen. So kann und muss ,Arbeit' danach gegliedert werden, für wen sie geleistet wurde (Hausarbeit, Lohnarbeit, Subsistenzwirtschaft). ,Nichtarbeit ' hingegen beinhaltet nicht in jedem Fall Freizeit im heutigen Verständnis, vielmehr wurde arbeitsfreie Zeit genutzt, um Besuche zu machen, die teilweise der Arbeitsorganisation dienten, sich an den Arbeitsort oder nach Hause zu begeben (Mobilität), zum Essen und Schlafen oder für den Kirchgang. Nichtsdestoweniger finden sich in dieser Kategorie aber auch die Untergruppen ,Spiel und Tanz', ,Wirtshaus' oder, Unzucht'.

35 Die Formulierung ,genau' muss in diesem Zusammenhang relativiert werden, so finden sich im Idealfall Angaben zu halben und Viertelstunden, Minutenangaben hingegen nur sehr selten.

36 Hermann Grotefend, Taschenbuch der Zeitrechnung des deutschen Mittelalters und der Neuzeit, Hannover 2007. 


\section{Hochrechnung der zu erwartenden Datenpunkte in den Archiven \\ Dresden und Leipzig}

Die aus der Stichprobe gewonnenen Erkenntnisse lassen Hochrechnungen über die Menge verfügbarer Datenpunkte in den Archiven Dresden und Leipzig in den unterschiedlichen Kategorien zu. In diese Berechnung mit einfließen müssen auch die Akten, aus denen keine Datenpunkte gewonnen werden konnten. Dies betrifft rund die Hälfte aller durchgesehenen Akten und relativiert den durchschnittlichen Ertrag an Datenpunkt je Akte deutlich. Am ergiebigsten waren die Delikte ,Brand und Brandstiftung', ,Leichenfund', ,Körperverletzung' und ,Mord'. Am unteren Ende der Tabelle findet sich die Kategorie ,Betrug‘.

Setzt man nun die Summe der Bestände in den Archiven Dresden und Leipzig in Verbindung mit der durchschnittlichen Datenpunkt-Menge je Akte, ergibt sich das in Tabelle 6 festgehaltene Bild. In Folge dieser Hochrechnung erhält man eine Summe von 7416 Datenpunkten für die bereits stichprobenartig untersuchten Kategorien. Ein weiteres großes Potenzial beinhalten die noch nicht untersuchten Bestände in Chemnitz und Bautzen.

\begin{tabular}{|c|c|c|c|c|c|c|c|}
\hline Delikt & $\begin{array}{l}\text { Akten } \\
\text { gesamt } \\
\text { (Akten } \\
\text { ohne } \\
\text { DP) }\end{array}$ & $\begin{array}{l}\text { Anzahl } \\
\text { DP } \\
\text { absolut }\end{array}$ & $\begin{array}{l}\text { Durch- } \\
\text { schnitt- } \\
\text { liche } \\
\text { Anzahl } \\
\text { DP je } \\
\text { Akte }\end{array}$ & $\begin{array}{l}\text { Durch- } \\
\text { schnitt- } \\
\text { liche } \\
\text { Anzahl } \\
\text {,harter } \\
\text { DP' } \\
\text { je Akte }\end{array}$ & $\begin{array}{l}\text { Durch- } \\
\text { schnitt- } \\
\text { liche } \\
\text { Anzahl } \\
\text {,weicher } \\
\text { DP' } \\
\text { je Akte }\end{array}$ & $\begin{array}{c}\text { Anzahl } \\
\text { Akten } \\
\text { (Dres- } \\
\text { den } \\
\text { und } \\
\text { Leip- } \\
\text { zig) } \\
\end{array}$ & $\begin{array}{l}\text { Schät- } \\
\text { zung } \\
\text { DP }\end{array}$ \\
\hline $\begin{array}{l}\text { Aufwiege- } \\
\text { lung, } \\
\text { Friedens- } \\
\text { störung, } \\
\text { Revolution }\end{array}$ & $8(6)$ & 4 & 0,5 & 0,2 & 0,3 & 893 & 447 \\
\hline Beleidigung & $10(8)$ & 5 & 0,5 & 0,1 & 0,4 & 446 & 223 \\
\hline $\begin{array}{l}\text { Betrug, } \\
\text { Glücksspiel, } \\
\text { Falschmün- } \\
\text { zerei }\end{array}$ & $4(3)$ & 3 & 0,75 & 0,5 & 0,25 & 357 & 267,8 \\
\hline $\begin{array}{l}\text { Brand, } \\
\text { Brandstif- } \\
\text { tung }\end{array}$ & $10(2)$ & 59 & 5,9 & 0,6 & 5,3 & 266 & 1570 \\
\hline $\begin{array}{l}\text { Diebstahl, } \\
\text { Einbruch, } \\
\text { Raub, } \\
\text { Überfall }\end{array}$ & $25(15)$ & 42 & 1,68 & 0,36 & 1,32 & 1106 & 1858,1 \\
\hline Konzession & $10(10)$ & 0 & 0 & 0 & 0 & 64 & 0 \\
\hline $\begin{array}{l}\text { Körperver- } \\
\text { letzung, } \\
\text { fahrlässige } \\
\text { Tötung, } \\
\text { Gewalt }\end{array}$ & $17(9)$ & 34 & 2 & 0,2 & 1,8 & 305 & 610 \\
\hline
\end{tabular}


Forschung und Diskussion

\begin{tabular}{|c|c|c|c|c|c|c|c|}
\hline $\begin{array}{l}\text { Leichen- } \\
\text { fund, } \\
\text { Selbstmord, } \\
\text { Todesfall }\end{array}$ & $22(9)$ & 122 & 5,54 & 0,9 & 4,64 & 88 & 487,5 \\
\hline $\begin{array}{l}\text { Mord, } \\
\text { Kindsmord, } \\
\text { Totschlag }\end{array}$ & $30(14)$ & 56 & 1,87 & 0,4 & 1,47 & 425 & 794,8 \\
\hline $\begin{array}{l}\text { Presse, } \\
\text { Zensur }\end{array}$ & $15(15)$ & 0 & 0 & 0 & 0 & 196 & 0 \\
\hline $\begin{array}{l}\text { Sittlichkeit } \\
\text { und unge- } \\
\text { bührliches } \\
\text { Verhalten }\end{array}$ & $13(7)$ & 18 & 1,38 & 0,55 & 0,83 & 418 & 576,8 \\
\hline Sonstiges & $9(1)$ & 10 & 1,1 & 0,45 & 0,65 & 528 & 581 \\
\hline Summe & & & & & & & 7416 \\
\hline
\end{tabular}

Tab. 6: Anzahl,harter' und,weicher' Datenpunkte (DP) je Akte und Schätzung der Datenpunkte aus dem Gesamtbestand der Archive Dresden und Leipzig unter Berücksichtigung unergiebiger Akten.

\section{Probleme und Fehler bei der Datenerhebung}

Aus den genannten Quellencharakteristika ergeben sich folgende Besonderheiten bei der Datenerhebung: Grundsätzlich kann vor Beginn der Beschäftigung mit der einzelnen Akte anhand der Informationen in den Findbüchern nicht ersehen werden, ob die entsprechenden Quellen Zeugenverhöre enthalten, wenn auch bestimmte Delikte ein Vorhandensein wahrscheinlicher werden lassen. Unumgänglich ist daher eine detaillierte und gründliche Sichtung, d. h. zunächst ein ,Fahnden“ nach Aussagen in jeder Akte. Nicht selten stellt sich dabei heraus, dass zu dem betreffenden Fall keine Zeugen gehört wurden. Daneben können aufgefundene Verhördaten aufgrund fehlender oder ungenauer Angaben, so wenn sich keine Verknüpfung zwischen einer Zeitangabe und einer Tätigkeit innerhalb der Aussage findet, nicht in das Datenset aufgenommen werden. Ähnlich gestaltet sich dies bei den Angaben zu den betreffenden Personen. Demgegenüber führt ein Zeugenbericht nicht selten zu mehr als einem Datenpunkt, z. B. wenn Aussagen zum Aufenthalt verschiedener Personen zu einer bestimmten Zeit an einem bestimmten Ort getätigt werden oder ein vollständiger Tagesablauf wiedergegeben wird. Positiv auf die Bearbeitungszeit wirkt sich nach dem Auffinden eines Verhörs aus, dass aufgrund der strukturellen Gemeinsamkeiten innerhalb der Quellengattung und der Verhörführung eine systematische Erfassung und Bearbeitung der Daten nach gleichbleibenden Kriterien erfolgen kann.

Aufgrund des erschwerten Auffindens der benötigten Informationen und des teilweise geringen Quellenertrages, muss ein erhöhter Zeitaufwand bei der Datenerhebung eingeplant werden. Es ist mit durchschnittlich 10 Minuten pro Datenpunkterhebung und Erfassung des relevanten Kontextes zu rechnen, wobei die Vorrecherche nicht eingeschlossen ist. 


\section{Quellenerträge}

Im Folgenden soll in einem ersten Versuch mithilfe unterschiedlicher Diagramme und Tabellen dargestellt werden, wie groß das Potenzial der Daten und wie vielfältig die sich daraus ergebenden Analyse- und Interpretationsmöglichkeiten sind. Die bisher erhobenen 274 Datenpunkte lassen bereits das Spektrum der unterschiedlichen Verknüpfungen von Zeit und Tätigkeit erahnen. Es wird deutlich, dass die in der historischen Forschung lange Zeit vernachlässigten Zeugenverhöre einen vielfältigen und nuancierten Informationsgehalt aufweisen, zumal diese Quellengattung bei der Beantwortung der Frage nach der Fleißrevolution innovative und tief greifende Möglichkeiten eröffnet, in die Alltagswelt einzelner Protagonisten vorzudringen. Trotz der noch geringen Anzahl an Datenpunkten kann bereits gezeigt werden, dass über den Untersuchungszeitraum Längsschnittdaten anhand unterschiedlichster Kriterien gebildet werden können. Es ist möglich, historische Zeitbudgets sowohl für den Tagesablauf als auch für die Strukturierung der Woche wie auch des gesamten Jahres für verschiedene soziale Schichten oder Berufsgruppen zu erstellen.

\begin{tabular}{cc}
\hline Johann Gottlieb Thate, Knecht, 20 Jahre & Johann Gottlieb Härtig, Großknecht, \\
27. Oktober 1733 (Sonntag) & 22 Jahre \\
& 14. Februar 1803 (Sonntag)
\end{tabular}

... am 26ten Octbr. 1807 abends um 8 Ubr Um 7 Ubr baben sämtliche Dienstleute habe ich diese Frauensperson zufällig auf gegessen, sind dann auch eine Weile in der dem Ratbskeller getroffen. Wir waren da Stube gestanden, haben dann das Vieb sehr vergnügt und tringen mit einander. gefüttert und sind nicht ... in die Stube zurückgekebrt.

Nach Verlauf einer Stunde geben wir nun Am Sonntag abend gleich nach 8 Ubr bin da zu dem Einwohner Breysing in ich mit Reinburgs Knecht und Schmidts Hartha, welcher ebenfalls zur Magd nach Miltitz zum Tanze gegangen Jahrmarktzeit Bier schenckt, dort halten und habe daselbst dem Tanze zugesehen. wir uns aber nicht lange auf...

Getanzt habe ich nicht, theils weil es so voll war theils, weil ich nicht viel tanze...

... sondern geben auf meine Veranlaßung Ich habe mich ... gebalten und, nachdem um halb $10 \mathrm{Ubr}$ Abends von da hinweg, $11 \mathrm{Ubr}$ vorbei gewesen, babe ich sie um uns in die Schenke nach Richze... aufgefordert, mit nach Lausen zu gehen ... ... daß undgefabr eine halbe Stunde von Die Entfernung von Miltitz bis Lausen Hartha entfernt ist, zu begeben. [...] weil mag ungefäbr eine halbe Stunde sein. der dortige Schankwirth Kirsten zum ... Jabrmarkte ebenfalls Musik und Tanz ...

... als ich nun nach Hause gehen wollen, ... in den Stall, wo ich schlafe, gegangen, in wäre Uhr 3 gewesen.

der Gutsherrnstube ... brannte noch Licht ... und welche Zeit es gewesen ist, weiß ich auch nicht, der ich keine Ubr fübre.

Tab. 7: Beispiele für Zeitbudgets. Quelle J.G. Thate: Acta Inquisitionis contra Johann Gottlieb Thaten, 1733-1735, Sächsisches Staatsarchiv - Staatsarchiv Leipzig, Amt Rochlitz 20017, Nr. 1916, fol. 11v-12r; Quelle J. G. Härtig: Acta das in der Nacht vom 17. zum 18. Febr. 1833 in dem Dorfe Lausen statt gefundene Feuer und einen in derselben Nacht daselbst verübten Geld-und Kleiderdiebstabl betr., 1833-1835, Sächsisches Staatsarchiv - Staatsarchiv Leipzig, Amt Leipzig 20009, Nr. 4367, fol. 17r-20r. 
Im Hinblick auf das Alter der aussagenden Personen können umfangreiche Schlussfolgerungen zu Beginn und Ende der Lebensarbeitszeit, Verschiebungen in diesem Bereich und den arbeitsintensivsten Phasen des Lebens getroffen werden. So kann bestimmt werden, ab welchem Alter Kinder in die Hausarbeit und spätere Erwerbsarbeit einbezogen wurden. Bereits auf Basis der erhobenen Daten lassen sich diesbezüglich einige Trends erkennen. Wie die folgende Grafik (Abb. 4) veranschaulicht, scheint sich die Lebensarbeitszeit im Laufe des Betrachtungszeitraums zu verlängern. Zum einen deutet sich an, dass sich die Einbeziehung von Kindern, mehrheitlich in die Hausarbeit und die Tätigkeiten der Subsistenz, intensiviert, zum anderen, dass Menschen zunehmend auch über das 60. Lebensjahr hinaus arbeiteten. Dies könnte einer zunehmenden Lebenserwartung, aber ebenso der Notwendigkeit einer Eigenversorgung auch im Alter geschuldet sein. Der Schwerpunkt der Lebensarbeitszeit scheint sich in der ersten Hälfte des 18. Jahrhunderts auf den Zeitraum zwischen dem 20. und 55. Lebensjahr, in der zweiten Hälfte auf das 12. bis 62. Lebensjahr erstreckt zu haben. Dabei muss die noch geringe Stichprobengröße berücksichtigt werden, doch offenbart die Darstellung, dass bei ausreichender Anzahl an Datenpunkten die Anwendung weiterer statistischer Analyseverfahren (beispielsweise Quantilregression) vielversprechende und signifikante Ergebnisse liefern kann.

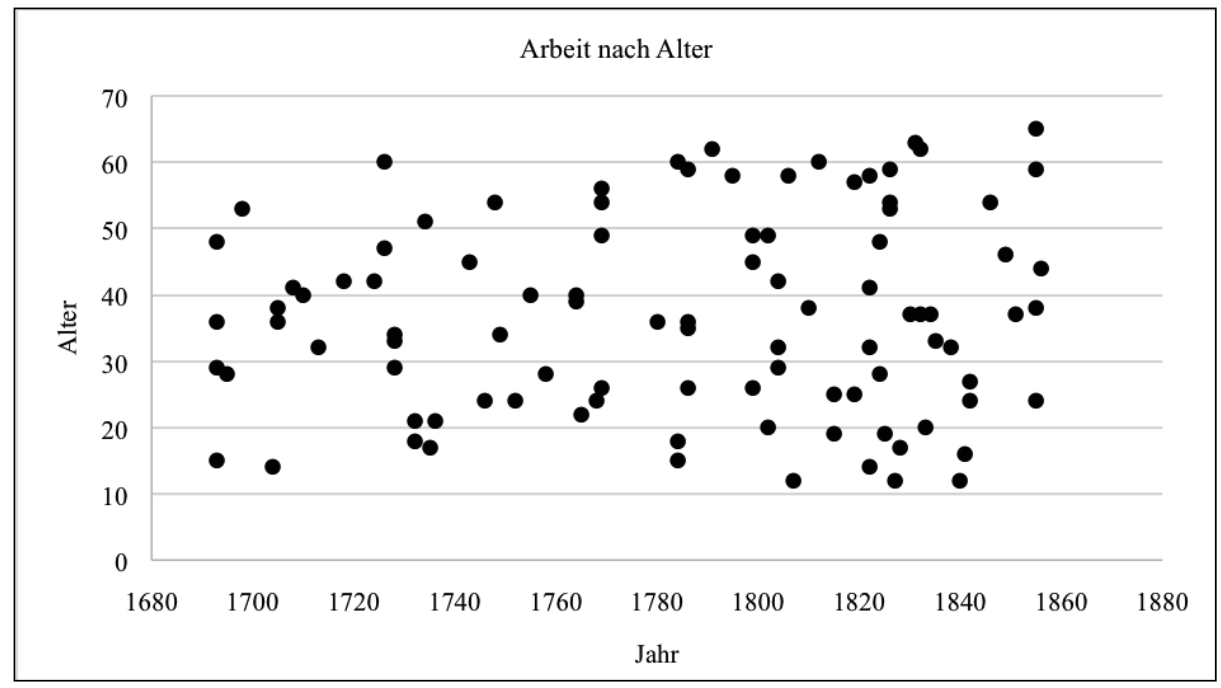

Abb. 4: Arbeit nach Alter.

Arbeit kann des Weiteren nach dem Geschlecht differenziert werden. Dies lässt Aussagen zur weiblichen und männlichen (Lebens-)Arbeitszeit zu, womit bei ausreichendem Stichprobenumfang u. a. die Frage beantwortet werden kann, ob Frauen durch Geburten ihre Tätigkeit unterbrachen oder trotzdem weiterhin aktiv an unterschiedlichen Arbeitsprozessen teilnahmen. Zudem ermöglichen die Datenpunkte zwischen Lohnarbeit, selbstständigen Tätigkeiten und Hausarbeiten zu differenzieren, d. h., es kann eine Antwort auf die Frage gefunden werden, wann ein Lohnarbeiter in die Arbeitswelt eintrat, wann der selbstständige Sohn eines Handwerkers oder ab welchem Alter die Unterstützung der Familie im jeweiligen Haushalt selbstverständlich dazugehörte. 
Neben Überlegungen zur (Lebens-)Arbeitszeit verschiedener Bevölkerungsgruppen lassen sich auch deutlich kleinere Analyseeinheiten bilden - und bereits die geringe Menge an Datenpunkten lässt Tendenzen und Verschiebungen ersichtlich werden. Zu diesen gehören Betrachtungen zur Verteilung von Arbeit über den Tag hinweg. An der Darstellung in Abbildung 5 ist erkennbar, dass Arbeit vermutlich insbesondere am Vormittag, d. h. zwischen 8 und 12 Uhr, geleistet wurde. ${ }^{37}$ Relativ konstant ist über den gesamten Zeitraum die Einhaltung der Mittagspause. Die Datenpunkte offenbaren, dass die Arbeitsintensität zwischen 12 Uhr und 13.30 Uhr deutlich geringer war, wie auch die Abendstunden (17 Uhr-22 Uhr) eher ein schwächeres Arbeitsmaß aufweisen. In der zweiten Hälfte des 18. Jahrhunderts scheint die Arbeitshäufigkeit am Nachmittag (13.30 Uhr-17 Uhr) und besonders in der Nacht (nach 22 Uhr) höher als noch zu Beginn des Jahrhunderts gewesen zu sein.

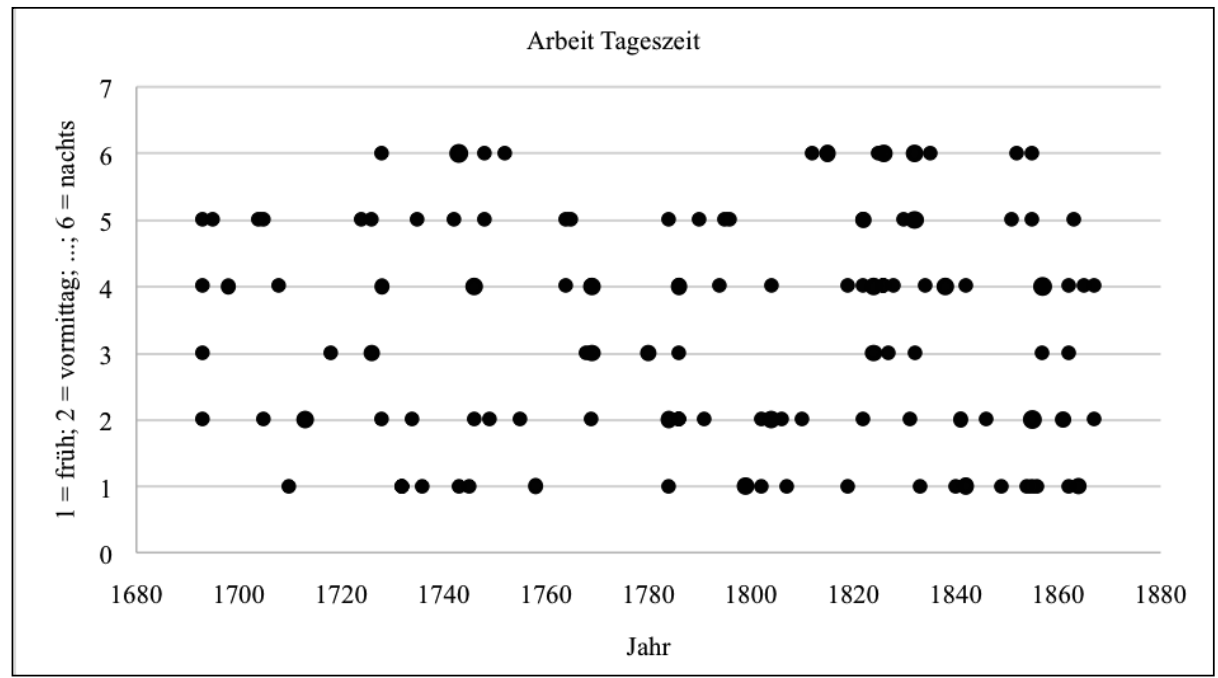

Abb. 5: Arbeit nach Tageszeit, $1=$ früh (5.00-8.00 Ubr); $2=$ vormittags $(8.00-12.00 \mathrm{Uhr}) ; 3=$ mittags $(12.00-13.30 \mathrm{Uhr}) ; 4=$ nachmittags $(13.30-17.00 \mathrm{Uhr}) ; 5=$ abends $(17.00-22.00 \mathrm{Uhr}) ; 6=$ nachts (22.00-5.00 $\mathrm{Uhr})$.

Deutlichere und bedeutungsvollere Aussagen zu Tendenzen erlaubt die Frage nach der Verteilung von Arbeit auf die Tage der Woche (im gesamten Untersuchungszeitraum), da eine größere Anzahl an Datenpunkten für diese Fragestellung herangezogen werden kann. Arbeitszeiten waren von 1690 bis 1870 keinesfalls gleichmäßig über die Woche verteilt, vielmehr können Tage herausgestellt werden, an denen anscheinend deutlich seltener beziehungsweise weniger gearbeitet wurde (Tab. 8). Hierbei wird jedoch noch nicht zwischen Hausarbeit, Lohnarbeit und selbstständiger Arbeit unterschieden.

Die arbeitszeitintensivsten Tage waren nach bisherigem Erkenntnisstand Dienstag und Freitag. Zieht man die Datenpunkte zur ,Nichtarbeit' hinzu, wird erkennbar, dass

37 Die Ermittlung der zeitlichen Zuschreibungen $\mathrm{zu}$ den verschiedenen Tageszeiten erfolgte anhand der Quellen und mithilfe von Aussagen, welche sowohl eine genau Zeit als auch eine Tageszeit angaben. Anhand dieser kann eine Umrechnung auch konkreter Zeiten in Tageszeiten vorgenommen werden. 
an den Tagen Montag, Mittwoch und Sonntag eine entsprechend größere Anzahl von Angaben zu alternativen Beschäftigungen gemacht wurden. Diese ersten explorativen Erkenntnisse verdeutlichen das Potenzial der Methode historischer Zeitbudgetermittlung und stellen gängige Vorstellungen zum ,Blauen Montag' als arbeitsfreien Tag der Handwerker in Frage. Mithilfe statistischer Analyseoperationen können auf größerer Datenbasis wichtige und belastbare Erkenntnisse zu dieser Frage und der nach Arbeitszeiten in der (Vor-)Moderne allgemein geliefert werden.

\begin{tabular}{lccc}
\hline Wochentag & $\begin{array}{c}\text { Arbeit } \\
\text { (prozentualer Anteil) }\end{array}$ & $\begin{array}{c}\text { Nichtarbeit } \\
\text { (prozentualer Anteil) }\end{array}$ & $\begin{array}{c}\text { Gesamtanzahl } \\
\text { Datenpunkte }\end{array}$ \\
\hline Montag & 42 & 58 & 36 \\
Dienstag & 58 & 42 & 31 \\
Mittwoch & 30 & 70 & 37 \\
Donnerstag & 46 & 54 & 26 \\
Freitag & 51 & 49 & 37 \\
Sonnabend & 48 & 52 & 33 \\
Sonntag & 44 & 56 & 36 \\
\hline
\end{tabular}

Tab. 8: Arbeit und Nichtarbeit nach Wochentagen.

Die erhobenen Datenpunkte lassen jedoch nicht nur Aussagen darüber zu, ob Menschen gearbeitet haben oder nicht, vielmehr gelingt es in vielen Fällen, Alltag zu rekonstruieren. So können detaillierte Angaben zur ,Freizeitgestaltung' beziehungsweise zur Beschäftigung in arbeitsfreien Phasen getroffen werden (Tab. 9). Anhand der Daten kann somit zum einen die Frage nach der Fleißrevolution in einem umfassenden Kontext beantwortet werden, zum anderen beinhalten sie ein großes Spektrum, das nicht nur Betrachtungen dazu ermöglicht, ob die Menschen im Laufe der Zeit mehr arbeiteten, sondern vielmehr auch in welchen Bereichen die Mehrarbeit geleistet wurde beziehungsweise was die Menschen zu unterschiedlichen Zeiten taten, wenn sie nicht arbeiteten und wie sie ihren Alltag strukturierten.

\begin{tabular}{lccccc}
\hline Wochentag & $\begin{array}{c}\text { Tanz } \\
\text { (prozen- } \\
\text { tualer } \\
\text { Anteil) }\end{array}$ & $\begin{array}{c}\text { Wirtshaus } \\
\text { (prozen- } \\
\text { tualer } \\
\text { Anteil) }\end{array}$ & $\begin{array}{c}\text { Unzucht } \\
\text { (prozen- } \\
\text { tualer } \\
\text { Anteil) }\end{array}$ & $\begin{array}{c}\text { Sonstiges } \\
\text { (prozen- } \\
\text { tualer } \\
\text { Anteil) }\end{array}$ & $\begin{array}{c}\text { Gesamtanzahl } \\
\text { Datenpunkte } \\
\text { Nichtarbeit }\end{array}$ \\
\hline Montag & 14 & 10 & 19 & 57 & 21 \\
Dienstag & 38 & 23 & 15 & 24 & 13 \\
Mittwoch & 23 & 12 & 23 & 42 & 26 \\
Donnerstag & 14 & 43 & 22 & 22 & 14 \\
Freitag & 28 & 39 & 11 & 22 & 18 \\
Sonnabend & 18 & 35 & 41 & 6 & 17 \\
Sonntag & 50 & 0 & 30 & 20 & 20 \\
\hline
\end{tabular}

Tab. 9: Aktivitäten nach Wochentagen. 


\section{Schlussbetrachtung}

Jan de Vries' These der Fleißrevolution als eine Voraussetzung für die europäische Industrialisierung ist in den letzten beiden Jahrzehnten mehrfach anhand unterschiedlicher Methoden und auch Quellen geprüft worden. Bei den ersten der innovativen Studien wurde jedoch die Aussagekraft der Ergebnisse angezweifelt, aufgrund einer jeweils einseitigen Quellenauswahl: De Vries wertet Inventarverzeichnisse aus, deren beobachtbare Veränderungen auch andere Ursachen als eine Fleißrevolution gehabt haben könnten. Hans-Joachim Voths Studie wird u. a. aufgrund der Beschränkung auf zwei Regionen und auf männliche Zeugen kritisiert - eine Schwäche im Datenset, die nicht mittels ergänzender Quellen ausgeglichen wird. Im Gegensatz dazu nutzen Gregory Clark und Ysbrand van der Werf für ihre Betrachtung von englischen Holzarbeitern und Dreschern eine breitere Quellenbasis und analysieren Arbeitsausstoß, Jahreslohn, Preisraten und Realeinkommen. ${ }^{38}$ Auch die Studien von Sheilagh Ogilvie, die versucht de Vries' Thesen auf die „latedeveloping economy“39 Württembergs anzuwenden, beziehen nicht nur Inventare, sondern auch normative Quellen wie beispielsweise Polizeiordnungen, Lohnlisten, Landesgesetze und Eheregister ein. Anhand der bisherigen Forschungsarbeiten ist offensichtlich geworden, dass weder Inventarverzeichnisse, noch Kriminalakten oder normative Quellen allein ausreichend sind, um die Frage der Fleißrevolution zufriedenstellend beantworten zu können. Dementsprechend beschränkt sich unser Projekt nicht auf Kriminalakten und den Versuch, die Schwächen dieser Quellengattung, wie in Kapitel I.3 erläutert, auszugleichen. Um der beschriebenen Problematik entgegenzuwirken, werden nicht nur weitere Quellen, sondern auch verschiedene quantitative Methoden eingesetzt: neben 1. der Analyse von Zeitbudgets anhand von Zeugenaussagen in Verhörprotokollen, 2. der Vergleich von Tages- und Jahreslöhnen beziehungsweise der Reallöhne, jedoch nicht nur von Lohnarbeitern wie in der Studie von Clark/van der Werf und 3. die Analyse der saisonalen Verteilung von Heiraten mittels Kirchenbüchern. Die Untersuchung strebt somit eine Erklärung makroökonomischer Prozesse mithilfe einer mikroökonomischen Studie an. Die Ergebnisse der quantitativen Analysen sollen mit Blick auf politik- und religionshistorische, ökonomische und demografische Prozesse interpretiert werden. Erforderlich ist hierfür die Rekonstruktion des liturgischen Kalenders Sachsens zwischen Reformationszeit und 19. Jahrhundert sowie der auf dessen Reform sowie auf die Arbeitsrollen und das Arbeitsverhalten von Männern, Frauen und Kindern bezogenen Politik des Landes. Wichtige erklärende Größen bilden weiter die Ausbildung von Produkt- und Konsummärkten, sowie die relativen Anteile von Arbeitsfähigen und Abhängigen an der Bevölkerung.

Das Projekt beabsichtigt, Daten zur Arbeitszeit zu erheben und diese mit der zunehmenden Bedeutung des Konsums in einen Zusammenhang zu stellen, um die Frage nach einer Fleißrevolution für Sachsen beantworten zu können. Dabei soll nicht nur ermittelt werden, ob der Einzelne durch mehr (Lohn-)Arbeit ein höheres Einkommen erwirtschaftete, um dadurch mehr Konsumgüter erwerben zu können, sondern auch, ob zu diesem Zweck im Laufe des Betrachtungszeitraums zunehmend über den eigenen Subsistenzbedarf hinaus gearbeitet wurde. Durch einen Stadt-Land-Vergleich kann die Hypothese überprüft werden, dass Städte und urbane Zentren aufgrund ihrer größeren Marktnähe eine Konsumrevolution initiierten, während ländliche Regionen

38 Clark/van der Werf, Work in Progress? (wie Anm. 3), S. 830-843.

39 Sheilagh Ogilvie, Consumption, social capital, and the ,Industrious revolution ' in early modern Germany, in: The journal of economic history 70 (2010), S. 287-325, hier 319. 
verspätet in diese Entwicklung eintraten. ${ }^{40}$ Sachsen eignet sich als Untersuchungsgebiet aufgrund einer guten Quellenüberlieferung historischer Kriminalakten. Diese erlaubt die gesamte Struktur des Landes durch die Berücksichtigung möglichst vieler lokaler, aber ebenso landesherrlicher Gerichtsinstanzen und die Erhebung landesweiter Daten für Stadt und Land zu erfassen. Die Studie kann somit weit über die punktuellen Erhebungen Voths für England hinausgehen. Sachsen empfiehlt sich weiterhin durch eine ähnliche wirtschaftliche Ausrichtung wie England (Bergbau und Textilindustrie). Zahlreiche sächsische Industrielle nahmen sich England zum Vorbild, besuchten dieses Land und brachten neue Impulse mit in ihre Heimat. Insofern stellt eine Analyse Kursachsens, mit einem indirekten Vergleich zu England, einen vielversprechenden Ansatz dar.

Trotz der durchaus zahlreichen Arbeiten zu Fragen und Zusammenhängen der Fleißrevolution in unterschiedlichen ökonomischen und institutionellen Gegebenheiten offenbart sich ein immer noch hoher Forschungsbedarf, insbesondere um eine Vergleichbarkeit der kontroversen Ergebnisse zu ermöglichen und die Bedingungen, Hintergründe und Voraussetzungen wie auch die Folgen einer Verfleißigung vor verschiedenen regionalen, konfessionellen und ökonomischen Hintergründen zu kontextualisieren. ${ }^{41}$ In diesem Umfang kann das Projekt einen entscheidenden Beitrag zur weiteren Erforschung einer früh industrialisierten deutschen Region liefern und schließt nahtlos an unterschiedliche Studien an, die sich bereits mit der wirtschaftlichen Sonderstellung Sachsens auseinandergesetzt haben. ${ }^{42}$ Darüber hinaus wird es

40 Dabei müssen jedoch auch die unterschiedlichen Einkommen und Berufsstrukturen zwischen städtischer und dörflicher Bevölkerung Berücksichtigung finden und nach den individuellen Merkmalen der Konsumenten gefragt werden. Beispiele für solche Analysen finden sich bei Mark Overton/Jane Whittle/Darron Dean/Andrew Hann, Production and Consumption in English Households, 1600-1750, London 2004, S. 157-169; Lorna SCAmmell, Town versus country: the property of every day consumption in the late seventeenth and early eighteenth centuries, in: Jon Stobart/ Alastair Owens (Hg.), Urban Fortunes. Property- and Inheritance in the Town, 17001900, Aldershot 2000, S. 35-49.

41 Vgl. Ogilvie, Consumption (wie Anm. 39).

42 Vgl. Rudolf Boch, Staat und Industrialisierung im Vormärz: Das Königreich Sachsen (mit Vergleich zu Preußen), in: Manfred Hettling (Hg.), Figuren und Strukturen. Historische Essays für Hartmut Zwahr zum 65. Geburtstag, München 2002, S. 355-371; Rudolf Forberger, Die Industrielle Revolution in Sachsen 1800-1861, 2 Bde., Berlin 1982; Ullrich Hess/Michael Schäfer/Petra Listewnik (Hg.), Wirtschaft und Staat in der Industrialisierung Sachsens 1750-1930, Leipzig 2003; Hubert KiesewetTer, Staat und Unternehmen während der Frühindustrialisierung. Das Königreich Sachsen als Paradigma, in: Zeitschrift für Unternehmensgeschichte 29 (1984), S. 1-32; Ders., Staat und regionale Industrialisierung. Württemberg und Sachsen im 19. Jahrhundert, in: Ders./Rainer Fremdling (Hg.), Region und Industrialisierung, Ostfildern 1985, S. 108-132; Ders., Die Industrialisierung Sachsens. Ein regional-vergleichendes Erklärungsmodell (Regionale Industrialisierung 5), Stuttgart 2007; Kopsidis/Pfister, Agricultural development (wie Anm. 26); Uwe Schirmer (Hg.), Sachsen 1763-1832. Zwischen Rétablissement und bürgerlichen Reformen (Schriften der RudolfKötzschke-Gesellschaft 3), Beucha 1996; Martin Uebele/Tim Grünebaum, Food security, harvest shocks, and the potato as secondary crop in Saxony, 1792-1811, in: Groningen Growth and Development Centre Research Memorandum 139 (2013), http://ggdc.eldoc.ub.rug.nl/FILES/root/WorkPap/2013/GD-139/gd139.pdf [Zugriff am 21. September 2014]; Martin Uebele/Tim Grünebaum/Michael Kopsidis, King's law and food storage in Saxony, c. 1790-1830, in: Center for Quantitative 
auch möglich sein, einen nach unterschiedlichen sozialen Gruppierungen differenzierten Einblick in die Sozialgeschichte des Alltags zu geben. Dabei können Antworten auf die Fragen gefunden werden, wie Menschen ihren (Arbeits-)Alltag organisierten und $\mathrm{zu}$ welchen Zeiten sowie aufgrund welcher Ursachen Änderungen erforderlich waren.

Economics. Working Papers 26 (2013), http://www1.wiwi.uni-muenster.de/cqe/forschung/publikationen/cqe-working-papers/CQE_WP_26_2013.pdf [Zugriff am 21. September 2014]. 